\title{
Dialectic of homochirality
}

\section{Alexander Kholmanskiy}

A.I. Yevdokimov Moscow State University of Medicine and Dentistry, Moscow, Russian, E-mail: allexhol@ya.ru, https://orcid.org/0000-0001-8738-0189

\begin{abstract}
To solve the problem of the origin of homochirality of living systems and life itself, it is necessary to rethink the conceptual foundations of physics dialectically. The review shows how this can be done within the framework of the paradigm of energoforms, which sets the principles of construction and mathematical formalization of the Heraclitus primary element of matter. The algorithm for modeling energoforms and structures of elementary particles was created based on the laws of dialectics, electromagnetic induction and the rules of quantum physics. The algorithm allowed to calculate the parameters of chiral structures of neutron, proton, electron, neutrino and photon in the ground and excited states, as well as the parameters of more than one hundred nuclei. As an adequate solution to the problems of neutrino physics and the nature of the universal chiral factor, we hypothesized the instability of the low-energy solar neutrino and the biogenicity of its decay products - chiral energoforms. The hypothesis was based on correlations of seasonal and daily variations of solar neutrino flux, optical activity of aqueous solutions of sugars and metabolism of plants and animals. In humans, chiral morphofunctional features that limit homeostasis and the rhythm of energy-informational connections of the brain with the external environment were revealed. It was shown that the mechanism of sensitivity of living matter to the action of chiral factor is based on the ability to self-organization of its cooperative watercontaining systems consisting of spin homogeneous chiral elements.
\end{abstract}

Keywords: homochirality; dialectics; energoform; chiral factor; neutrino; biophysics. 


\section{Graphic abstract}

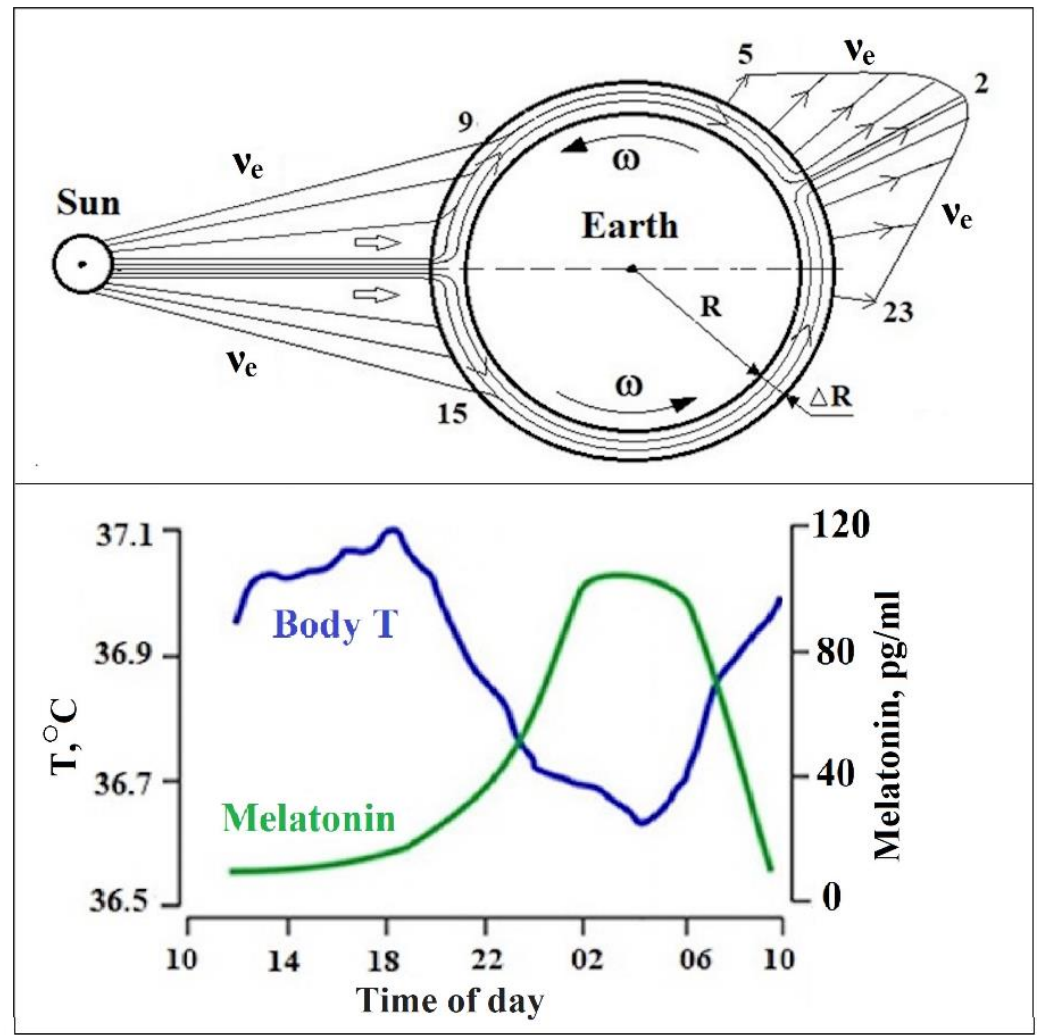

\section{Introduction}

Our theoretical tools ... are becoming inadequate to address the next layer of open questions. A new paradigm change seems to be necessary.

Gian Francesco Giudice [1] By virtue of the spirit of scientific thinking, birth truly new scientific advances impossible without interaction with philosophical ideas. Werner Heisenberg [2].

The natural philosophy of the homochirality of living systems combines the still unsolved problems of the physicochemical mechanisms of the synthesis of chiral organic molecules at the prebiotic stage of evolution and the subsequent chiral differentiation of ontogenesis and metabolism of living organisms. Living systems are open systems, the thermodynamics of which largely depends on the anomalous properties of liquid water, both external and intrasystem [3, 4]. Since homochirality is an exceptional feature of living systems, the factor determining its genesis should also be significant for the concept of the Anthropic principle, as well as the fundamental 
laws of physics. It can be assumed that the physical nature of this chiral factor $(\mathrm{CF})$ is identical to the nature of elementary particles and is universal, which implies the possibility of the participation of $\mathrm{CF}$ in the genesis of the mechanism of heuristic thinking of homo sapiens.

Thus, the solution to the homochirality problem is limited by the degree of adequacy of elementary particle physics (EP), currently the Standard Model. This model is still far from completion, as evidenced by the recognition of theoretical physicists: we strongly misunderstand how the world of elementary particles works. The standard model of physics is not complete - for example, the existence of dark matter and the presence of mass in neutrinos go beyond its limits [5]. Note that the dialectic flaw in the concept of point and chiral sterile EP excludes the possibility of receiving an answer to the question: why is there a substance, but there is no antimatter [5]. For the same reason, outside of physics, the problems that arise when considering the ordering of biological structures, its creation and evolution, do not lie in the field of physics [6]. The solution of these problems is associated with the establishment of the nature of $\mathrm{CF}$, which is responsible for the emergence of homochirality and the subsequent development of dissymmetry of living systems [7-14]. Recently, a number of hypothetical models of chiral symmetry breaking during the synthesis or destruction of the simplest amines and sugars at the protoplanetary stage of the Solar System formation [15] or in space under the action of supernova neutrinos [16-21], polarized photons with energy from $\sim 0.5$ to $\sim 7 \mathrm{eV}$ [22 - 26] and electrons [12, 27-29], as well as with the participation of the magnetic field of neutron stars [20, 21]). In these models, different compositions of compounds were proposed as a substrate: $\mathrm{H}_{2} \mathrm{O}$ (ice), $\mathrm{NH}_{3}, \mathrm{CHN}_{,} \mathrm{CH}_{4}, \mathrm{CH}_{3} \mathrm{OH}$, CO. In [15], reaction schemes were modeled in the conditions of a primitive atmosphere/hydrosphere or on granules of gas-dust clouds. It was assumed that metal catalysts could participate in the synthesis of right sugar in the hydrosphere and its yield increased the effect of autocatalysis.

The violation of the chiral symmetry of amines in the Supernova Neutrino Amino Acid Processing (SNAAP) model [19-21] is associated with the predominance of destruction of right amines over left ones on meteorites exposed to a supernova antineutrino in a magnetic field of a neutron star. The unrealistic SNAAP model is indicated by the figure in it of a rotating meteorite, a neutron stars, whose radiation at a distance of 0.01-0.05 AU [20] does not leave traces of ice, amines and volatile substrate compounds on the meteorite. To this should be added the negligible probability of the capture of high-energy antineutrinos with ${ }^{14} \mathrm{~N}$ nitrogen $[30,31]$ and the formation 
of ${ }^{14} \mathrm{C}$. The nearest neutron star is about 400 ly distant from the Earth, so a meteorite flying at a speed of about $30 \mathrm{~km} / \mathrm{s}$ will cover this distance in $\sim 4$ million years. During this time, with a halflife of ${ }^{14} \mathrm{C} \sim 5700$ years, the concentration of ${ }^{14} \mathrm{~N}$ and the racemic mixture of amines will fully recover on the meteorite.

The results of photolysis and radiolysis in the laboratory of various compositions of compounds of the substrate and with the addition of transition metals as catalysts showed the fundamental possibility of the formation of simple amines [12, 13, 26] and sugars [15, 22, 23]. However, due to the complexity of quantitative measurements and the impossibility of accurately reproducing natural conditions, laboratory results should be extrapolated with care to real processes. For example, in [32] it was noted that with an accurate description of an analog experiment in [22], most of the products could have been formed during their analysis, and not in the mother ice.

In almost all theoretical models of homochirality, the CF of a cosmogenic nature appears, acting at the initial prebiotic stage of evolution. However, life on Earth is unique on the scale of our Galaxy and this uniqueness is mainly due to the geochemistry of the Earth and the peculiarities of its physical connections with the Sun and the planets. Accordingly, the etiology of CF to a large extent should be associated with the physics of the Earth and the Sun, and its relevance should be maintained in one form or another at all stages of evolution. An analysis of the possible forms of realization of the $\mathrm{CF}$ solar-terrestrial nature was carried out in this review, taking into account the problems and reliable data of fundamental physics.

\section{Dialectics in physics}

Chirality and homochirality, in essence, are a manifestation at the level of living systems of the basic law of dialectics - the law of unity and struggle of opposites (hereinafter - the first LD). Heraclitus formulated this law 2500 years ago, presenting the fundamental principle of matter in the form of a dichotomous pair of essentially identical primary elements. The first LD is presented in physics in the form of Newton's third law (Action is always an equal and opposite reaction), the law of electromagnetic induction, the principle of least action by Hamilton and $a$ quantum of action equal to Planck's constant (h).

The physical nature of Heraclitus' discrete primary elements is in principle unknowable, and it can only be correlated with Kant's thing-in-itself. For the formalization of the chiral dichotomy of fundamental physics, the Adorno negative dialectic logic is applicable [33]. 
According to Adorno, the falsity of systematic knowledge that cannot reach the essence of things should be overcome with the help of non-systemic thinking, which preserves the independence of the true meaning of the subject from the methods of cognition of reality that are accessible and accessible to logical thinking. According to Heisenberg, such a rethinking of the fundamentals of physics is associated with a change in the very structure of thinking [2].

These ideas of Heisenberg and Adorno anticipated and implemented Maxwell in the formalization of the theory of the electromagnetic field and the dialectic of the ether: The fundamental dynamic idea of matter capable, thanks to its movement, becomes a reservoir of momentum and energy, so intertwined with our forms of thinking ... There is some ethereal medium filling the space and permeating all bodies. ... the environment has the ability to receive and save two types of energy, namely "actual" energy, depending on the movement of its parts, and potential energy, which is the work that the environment will perform due to its elasticity [34].

The author of this review, relying on the logic of the first law of dialectics and Maxwell's ideas about ether and molecular vortices [34], in [35] called Heraclite's primary element of matter the energoform (EF) and formulated the main provisions of the energy form paradigm (PEF). In subsequent papers [7, 36-41], the heuristic potential of a dialectical PEF was revealed using the example of calculating vortex models of elementary particles and nuclei, as well as modeling brain physics. Physicists and biologists are aware that to solve the problems of elementary particles [1, $5,42]$ and explain the origin of life and thinking [43], it is necessary to update and deepen theoretical tools to the level of PE: Structuring process: it is a spontaneous generation of ordered sequence, hierarchy of dynamic structures, or "energoforms". Their difference from the usual stable, static structures is that they are not "states", but "modes", and therefore not feasible separately, separately from the process [44]. Dimensionality of brain operations is beyond the scope of our ideas. ... the main thing in brain activity is not a regulating, but a constructive function. The brain primarily builds concepts and variants of external reality [45]. "Concepts" or thought forms can be represented as correlated ensembles of EF [41].

The interactions of the EF between themselves and their self-assembly into the structures of the EP and nuclei obey the rules of symmetry and two other fundamental laws of dialectics the law of similarity (second LD) and the law of transition of quantitative changes into qualitative (third LD). The second LD allows one to extrapolate to the level of the EF the laws of the dynamics of continuous media and the quantization rules acting at the atomic-molecular level: The Universe, 
which narrowed to the size occupied by an atom, would represent the same picture. The simplicity of the laws of nature allows us to observe and learn only relationships [46]; subtle similarities of things [47]. The third LD determines the necessary and sufficient number of elements of one level of the order of the world for them to acquire the quality of the next level of order. This number can be called a bootstrap quantum. The order of the structural organization of the world, similar to [36], was divided into the following qualitatively different levels: energoforms (I); EP, atoms, molecules (II); real world (III), stellar-planetary systems (IV), Universe (V). Below with examples it will be shown that the transitions between these levels satisfy the third LD with a bootstrap quantum equal to the Avogadro number $\left(\mathrm{N}_{\mathrm{o}}\right)$. With this in mind, the number $\mathrm{N}_{\mathrm{o}}$ is included in a series of world constants - the speed of light $(\mathbf{C})$, the unit charge $(\mathbf{e})$, and $\mathbf{h}$, which ensure the dialectic and physicochemical bylaws of the Anthropic principle.

\section{Chiral dichotomy of energetic forms and elementary particles}

The simplest models of the right and left EF, and their paired combinations are presented in Figure 1. Self-movement EF is quantitatively characterized by impulse (P) and angular momentum (L). The quantum of action of an EF is expressed through the products of three pairs of physical quantities - energy and its time of action (Et), impulse and "step" of translational motion (P $2 \pi r$ ), angular momentum and turnover by $2 \pi(\mathrm{L} 2 \pi)$. The mathematical formalism of the motion of a ring vortex in a continuous liquid medium is suitable for describing the motion of the v/g-EF: The vortex ring with its atmosphere moves in a continuous liquid medium as an independent liquid body and carries the whole force of the impulse that generated it [48]. The atmosphere of such a vortex and $v / \mathrm{g}-\mathrm{EF}$ will correspond to the Kelvin oval [49] and its momentum will be identical to the vortex momentum.

The vortex elements of the structures of the EP and nuclei (Figure 2) were built from charge-mass pairs of m/e-pair (Figure 1) in the form of a spheroidal shell (Sh) and toroidal orbitals - internal (In) and external (Ex). The condition of the integrity of the vortex structure of EF, EP, and the nucleus was expressed by the equation:

$$
w_{i} r_{i}=\text { const }
$$

$w_{i}$ is the angular velocity, $r_{i}$ is the radius of the element.

The elements of particles and nuclei formed from $\mathrm{m} / \mathrm{e}$-pair had a rest mass $\left(\mathrm{m}_{\mathrm{i}}\right)$, electric charge $\left(\mathrm{q}_{\mathrm{i}}\right)$, magnetic moment $\left(\mu_{\mathrm{i}}\right)$. The consistency of these quantities with each other and the angular momentum $\left(\mathrm{L}_{\mathrm{i}}\right)$ was expressed by the relation: 


$$
\mu_{i}=\sum \frac{L_{i}\left|q_{i}\right|}{2 m_{i} C}
$$

The values of L are quantized as follows: $\pm \mathrm{k} \hbar / 2$ (shell) and $\pm \mathrm{k \hbar}$ (orbitals), where $\mathrm{k}=1,2,3$ are the levels of excitation. The angular momentum and angular velocity determine the kinetic energy of rotation of the g-vortex $\left(E_{g}\right)$ in the composition $v / g-E F W_{j}=1 / 2 \mathrm{Lw}=1 / 2 \mathrm{Jw}^{2}, \mathrm{~J}$ is the moment of inertia of the torus-ring or torus-sphere (Figure 2). The total energy of the resting g-vortex, taking into account the virial theorem, was equated to $3 \mathrm{~W}_{\mathrm{j}}$. By analogy with a photon for a moving g-vortex, an equivalent mass $\left(\mathrm{m}_{\mathrm{g}}\right)$ was introduced and the corresponding energy $\mathrm{m}_{\mathrm{g}} \mathrm{C}^{2}$ was equated to the total energy of motion $v / g-E F$ :

$$
E_{g}=\frac{1}{2} m_{g} C^{2}+\frac{3}{2} \sum L_{i} w_{i}=m_{g} C^{2},
$$

here are summarized $\mathrm{W}_{\mathrm{j}}$ shells and orbitals. The $v$-vortex models the rotational motion of a substance and its environment in planes perpendicular to the axes of the torus or the shell. Its energy $E_{v}$ is expressed by the formulas $[7,36]$ :

$$
\mathrm{E}_{v}=\mathrm{PC}=\hbar \mathrm{C} / \mathrm{r},
$$

here $\mathrm{r}$ is the radius of the torus section (Figure 1).

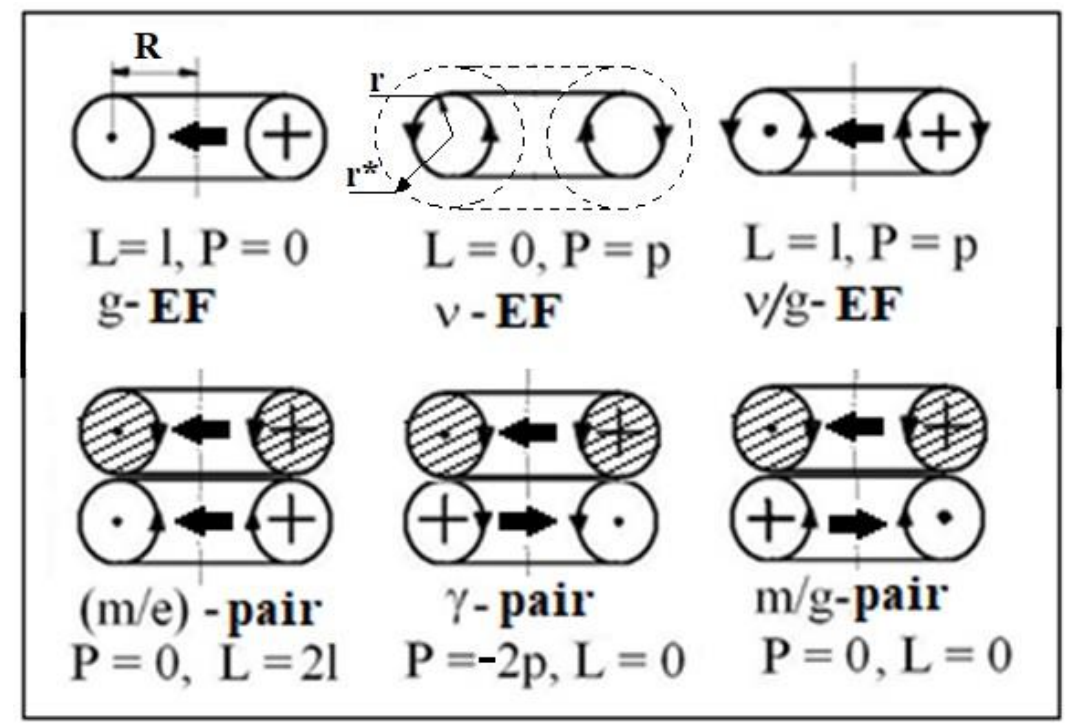

Figure 1. Vortex energoform models. $P$ - impulse, L - moment of impulse, left vortices shaded: $g$ $E F$ - rotating element of the ether; $v$-EF - an element moving progressively; $v / g-E F$ - the element is moving rotationally-progressively; $\gamma$-pair - self-moving element of the photon, neutrino; $m / e$ pair is a charge-mass element of particles with a rest mass (neutron, proton, electron); $m / g$-pair element of the relic ether (dark matter); from [7].

The first law of dialectics at the EF level was mathematically formalized, expressing the energy $E_{v}$ by real numbers, and the energy $E_{g}$ by imaginary numbers. Then the total energy of the 
ESP corresponds to a complex number:

$$
\mathrm{E}=\mathrm{PC}+\mathrm{img}_{\mathrm{g}} \mathrm{C}^{2}
$$

The dichotomy of translational and rotational motion of EF was formalized, by equating to zero the module of its energy:

$$
|\mathrm{E}|^{2}=\mathrm{EE}^{*}=\left(\mathrm{PC}+\mathrm{im}_{\mathrm{g}} \mathrm{C}^{2}\right)\left(\mathrm{PC}-\mathrm{im}_{\mathrm{g}} \mathrm{C}^{2}\right)=(\mathrm{PC})^{2}+\left(\mathrm{m}_{\mathrm{g}} \mathrm{C}^{2}\right)^{2} \equiv 0 .
$$

The quantization of changes in $\mathrm{E}_{v} v / \mathrm{g}-\mathrm{EF}$, as well as the atmospheres of EP and nuclei, for example, in an accelerator, describes the equation:

$$
\mathrm{N} \hbar \mathrm{C} / \mathrm{r}^{*} \leftrightarrow \hbar \mathrm{C} / \mathrm{r},
$$

The direct process in (1) represents the condensation of $\mathrm{N} v$-EF with radius $\mathrm{r}^{*}=\mathrm{Nr}$ into one $v$-EF with radius $r$ (Figure 1). The reverse process in (2) models the process of emitting a quantum of energy and is called unwinding.

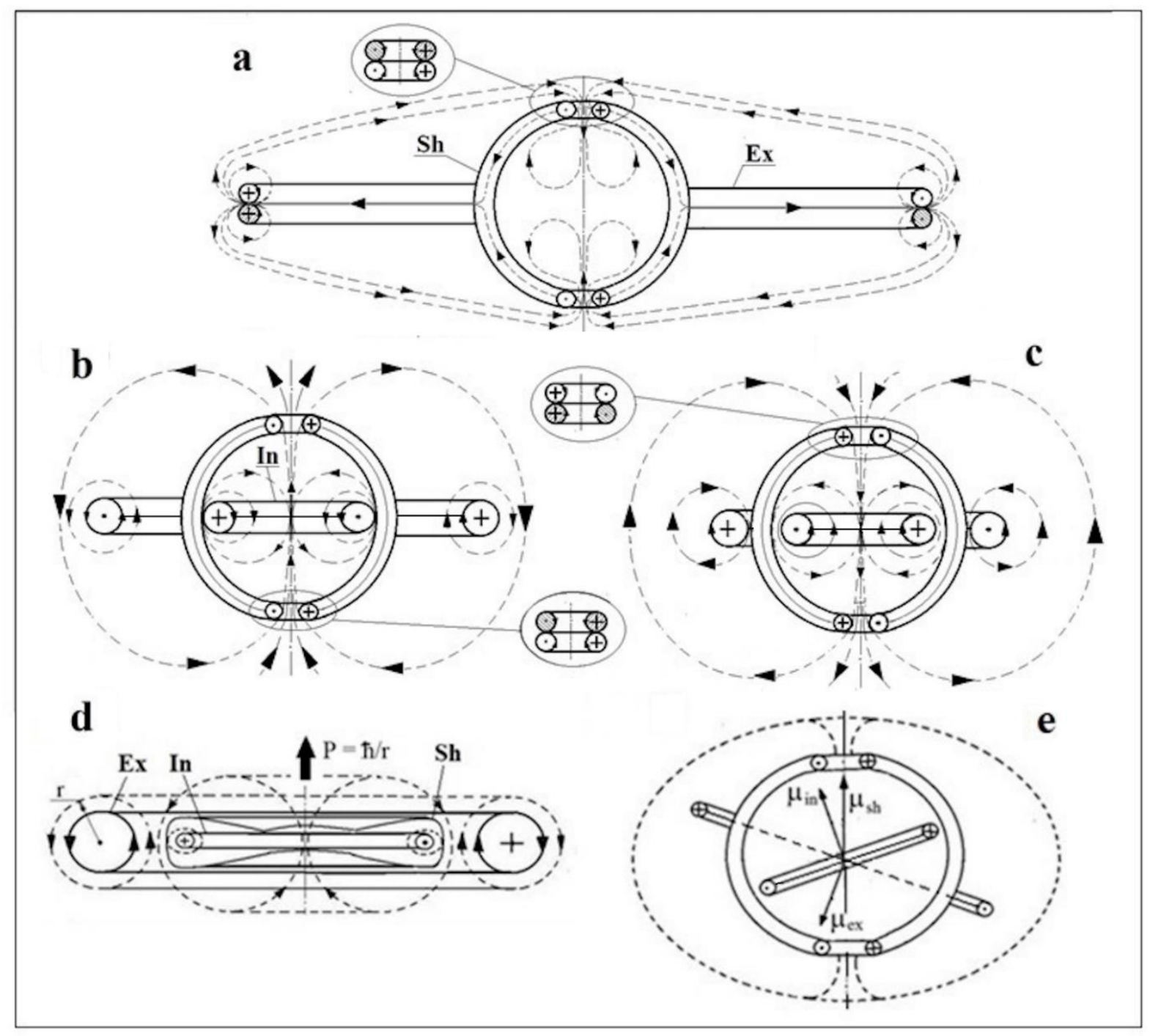


Figure 2. Models of the structure of the neutron (a), proton (b), electron (c), relativistic proton $(d)$ and the open shell of the structure of the nucleus $(e)$. The dotted line and arrows show the vortex flows of v-vortices, forming an atmosphere of particles and nuclei. Sh - shell, Ex, In external and internal orbital; $\mu_{i}$ are the magnetic moments of the elements; $r$ is the radius of the $v$-EF responsible for the particle momentum $(P)$ from [38-40].

When calculating the radii, masses and angular velocities (w) of the EP vortex elements $[38,40]$ and nuclei [39], empirical values of their characteristics (charge, spin, magnetic moment, rest mass) were used as boundary conditions. The calculated parameters of the neutron, proton and electron in the ground state and in the lowest excited states $(\mathrm{k}=1,2)$ are given in the Table. The values of the radii and charges of Sh, In, Ex for nucleons correlated with experimental data on their sizes and charge distribution [38].

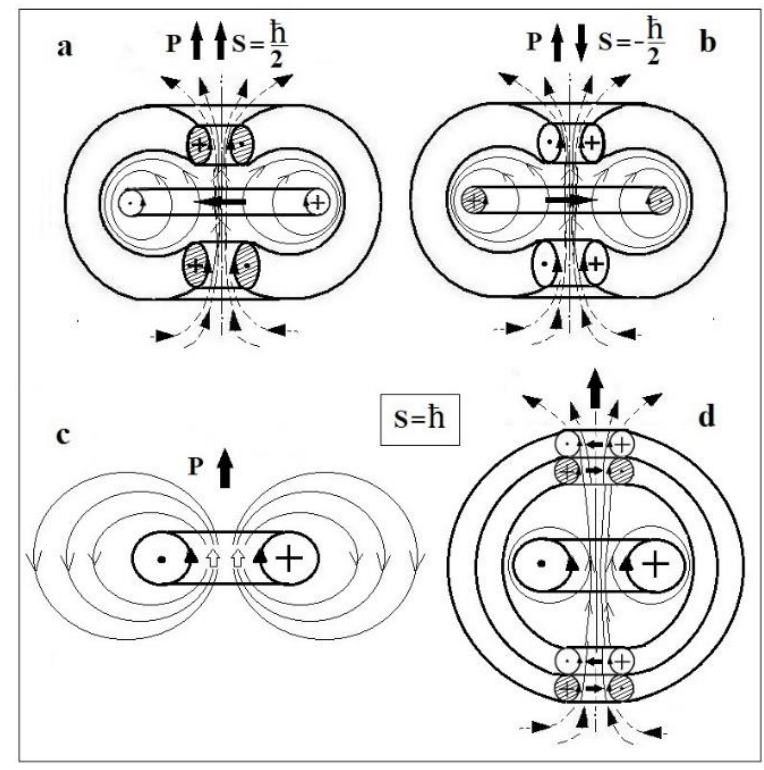

Figure 3. Vortex models of a right-handed antineutrino (a), a left-handed neutrino $(b)$ and a right-handed photon $(c, d), P$ - momentum, $S$ - spin; thin lines with arrows v-vortex flows. Shaded left spiral $v / g-E F$.

The simplest versions of the models of the electron neutrino $\left(v_{\mathrm{e}}\right)$, antineutrino $\left(\tilde{v}_{\mathrm{e}}\right)$ and photon $(\gamma)$ were built from self-moving $v / g$-EF and $\gamma$-pair (Figure 1) taking into account their spins and helicity signs [50]. When calculating the neutrino models, the values of the rest mass, charge, and magnetic moment were assumed to be zero. The excited states of $v_{\mathrm{e}}$ are called muon $\left(v_{\mu}\right)$ and tau $\left(v_{\tau}\right)$ neutrinos (Table). Radio waves and photons from the IR to the UV range simulate the structure (C) in Figure 3, and X-rays and gamma photons - a particle like structure (d).

When a particle absorbs a photon or quantum of EM energy in an accelerator or in the 
course of a nuclear reaction, the $v$-EF of a photon is embedded in the particle's atmosphere, giving it an additional impulse, and its equivalent mass g-EF increases the mass of the excited particle elements and this is formalized by increasing the number $\mathrm{k}$. In relativistic particles and nuclei, photon $v$-Efs as it were encapsulate their atmosphere (Figure 2e), which leads to a significant change in their properties. The lifetime of an unstable particle increases and the nature of its interaction with the substance changes $[40,51]$. The weak encapsulation of thermal neutrons explains the high cross-sections of their capture by nuclei, the strong encapsulation justifies the negligible probability of decay and inelastic interactions of highly energetic cosmic and reactor neutrinos with nuclei and particles [51].

Table

Parameters of particle models in the ground and excited states from [38]

\begin{tabular}{|c|c|c|c|c|c|c|c|c|c|}
\hline Particle & & $\begin{array}{l}\text { designation, } \\
\text { aths), elemer }\end{array}$ & ecay & $\begin{array}{l}\mathrm{L} \\
\text { (h) }\end{array}$ & $\underset{\text { (unit e) }}{\mathrm{q}}$ & $\underset{\text { (share) }}{\mathrm{m}}$ & $\begin{array}{c}\mathrm{w} \cdot 10^{-23} \\
\left(\mathrm{~s}^{-1}\right)\end{array}$ & $\begin{array}{c}\mathrm{r} \\
(\mathrm{Fm})\end{array}$ & $\begin{array}{l}\text { Life time } \\
\text { (s) }\end{array}$ \\
\hline \multirow{9}{*}{ Electron } & \multirow{3}{*}{0} & \multirow{3}{*}{ e } & Sh & $-1 / 2$ & $-2 / 3$ & 0.27 & $-2.0210^{-3}$ & $1.2710^{3}$ & \multirow{3}{*}{-} \\
\hline & & & In & -1 & $-2 / 3$ & 0.38 & $-2.1810^{-3}$ & $1.1710^{3}$ & \\
\hline & & & Ex & 1 & $1 / 3$ & 0.35 & $2,0210^{-3}$ & $1.2710^{3}$ & \\
\hline & \multirow{3}{*}{1} & \multirow{3}{*}{$\mu^{-}\left(e V_{\mu} V_{e}\right)$} & $\mathrm{Sh}$ & $-1 / 2$ & $-2 / 3$ & 0.16 & -0.24 & 10.6 & \multirow{3}{*}{$2.210^{-6}$} \\
\hline & & & In & -2 & $-2 / 3$ & 0.42 & -0.24 & 10.6 & \\
\hline & & & Ex & 2 & $1 / 3$ & 0.42 & 0.24 & 10.7 & \\
\hline & \multirow{3}{*}{2} & \multirow{3}{*}{$\pi^{-}\left(\mu^{-} v_{\mu} V_{e}\right)$} & Sh & -1 & $-2 / 3$ & 0.39 & -0.42 & 6.3 & \multirow{3}{*}{$2.610^{-8}$} \\
\hline & & & In & -2 & $-2 / 3$ & 0.51 & -0.42 & 6.3 & \\
\hline & & & Ex & 2 & $1 / 3$ & 0.10 & 0.08 & 31.0 & \\
\hline \multirow{6}{*}{ Neutron } & \multirow{2}{*}{0} & \multirow{2}{*}{$n$} & $\mathrm{Sh}$ & $1 / 2$ & $2 / 3$ & 0.72 & 12 & 0.24 & \multirow{2}{*}{$\sim 10^{3}$} \\
\hline & & & Ex & -1 & $-2 / 3$ & 0.28 & -35 & 0.80 & \\
\hline & \multirow{2}{*}{1} & \multirow{2}{*}{$\begin{array}{c}\Lambda(p \pi- \\
\left.n \pi^{\circ}\right)\end{array}$} & Sh & $1 / 2$ & $2 / 3$ & 0.51 & 3.2 & 0.84 & \multirow{2}{*}{$2.610^{-10}$} \\
\hline & & & Ex & -2 & $-2 / 3$ & 0.49 & $-3,5$ & 0.78 & \\
\hline & \multirow{2}{*}{2} & \multirow{2}{*}{$\Sigma\left(n \pi^{-}\right)$} & Sh & $3 / 2$ & $2 / 3$ & 0.45 & 2.9 & 0.93 & \multirow{2}{*}{$1.510^{-10}$} \\
\hline & & & Ex & -2 & $-2,3$ & 0.55 & $-3,9$ & 0.68 & \\
\hline \multirow{6}{*}{ Proton } & \multirow{3}{*}{0} & \multirow{3}{*}{ p } & Sh & $1 / 2$ & $2 / 3$ & 0.32 & 4.5 & 0.58 & \multirow{3}{*}{ - } \\
\hline & & & In & -1 & $-1 / 3$ & 0.42 & $-2 / 3$ & 0.58 & \\
\hline & & & Ex & 1 & $2 / 3$ & 0.28 & 2.8 & 0.93 & \\
\hline & \multirow{3}{*}{1} & \multirow{3}{*}{$\begin{array}{c}\Sigma^{+}\left(p \pi^{\circ}\right. \\
\left.n \pi^{+}\right)\end{array}$} & $\mathrm{Sh}$ & $1 / 2$ & $2 / 3$ & 0.19 & 12.4 & 0.39 & \multirow{3}{*}{$0.810^{-10}$} \\
\hline & & & In & -2 & $-2 / 3$ & 0.50 & -12.4 & 0.39 & \\
\hline & & & Ex & -2 & $1 / 3$ & 0.31 & 7.7 & 0.63 & \\
\hline
\end{tabular}

The core structure model is constructed taking into account the sign and magnitude of the quadrupole nuclear moment. It includes a system of enclosed enclosed shells formed from pairs of

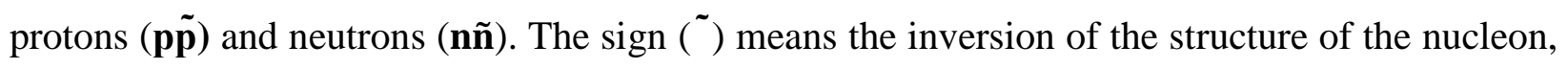


while the radii of related elements are preserved, and the spins are inverted. The charge of the pair

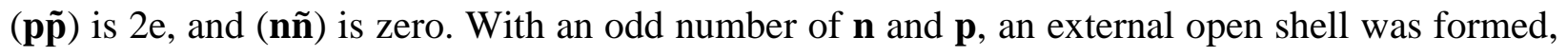
similar to the structure of a proton, neutron, or deuteron (Figure 2). The dependence of the calculated radii of nuclei and their isotopes on the mass number A for nuclei with $\mathrm{Z}$ from 1 to 37 (122 nuclei) correlated well with the empirical dependence of the radius on $(\mathrm{A})^{1 / 3}$ and a correlation was also observed between the radii of the nucleus and the atom of the element on $\mathrm{Z}[39$ ]. The latter result allows the possibility of the influence of the structure of the nucleus on the formation of atomic orbitals of electrons.

The dichotomy at the level of EP (n, p,e) and nuclei with nonzero spins and magnetic moments was expressed in the opposite direction of spins, magnetic moments and $v$-EF fluxes simulating the lines of force of nuclear and electromagnetic fields.

\section{Self-organization of the chiral universe}

In constructing the natural-philosophical model of the self-organization of the chiral Universe, the temperature anisotropy of the frequency spectrum of the cosmic microwave background (relic radiation) was taken into account (Figure 4) [52, 53]. The philosophy of the model was limited to the metaphysics of a continuous substance, from which in the act of dichotomous bifurcation a sufficient number of $v / g-E F$ quartets were formed, including such combinations of spin (s) and impulse (p): (p, s), (p, -s), ( -p, s), (-p, -s) [36]. The plus sign of the impulse and the back signifies their direction from bottom to top (Figure 1). The following stages of the model already have physical analogies (e.g. phase transitions, dew loss, Cooper pairs) and are consistent with the observed parameters of the particles and the Universe. Correlated condensation of the corresponding $\mathrm{v} / \mathrm{g}$-EF into elements of the shells and orbitals of neutrons that form a flat monolayer with the radius of the Universe $\left(\mathrm{R}_{\mathrm{Un}}\right)$ belongs to the stage of conditional fusion of EF. In the monolayer, neutrons with spins $\pm \mathrm{S}$ are staggered. With $\beta$-decay of neutrons, reactions of nuclear chemical synthesis, annihilation of nucleons, and electron-positrons competed. As a result, in $\sim 15$ min the monolayer turned into a plasma disk saturated with photons, antineutrino and containing nuclei and atoms of light elements up to oxygen. The distribution of light elements in the Universe $(\mathrm{H}-75 \% ; \mathrm{He}-23 \% ; \mathrm{O}-1 \% ; \mathrm{C}-0.5 \% ; \mathrm{N}-0.1 \%$ and $0.5 \%$ - the rest) was determined by the dominance of protons in the disk and the termination of nuclear fusion reactions on the He nucleus [39]. The disk could also be synthesized molecules $\mathrm{H}_{2}, \mathrm{OH}, \mathrm{H}_{2} \mathrm{O}, \mathrm{CH}$, $\mathrm{CO}, \mathrm{CN}, \mathrm{HCN}, \mathrm{NH}_{3}, \mathrm{H}_{2} \mathrm{CO}$, the sequence of which reflects a decrease in their content and 
correlates with the distribution and chemical activity of elements in the disk. The distribution in the interstellar medium of dipole molecules from this series correlates with the distribution of the relic radiation emitted by them (Figure 4) [53, 54].

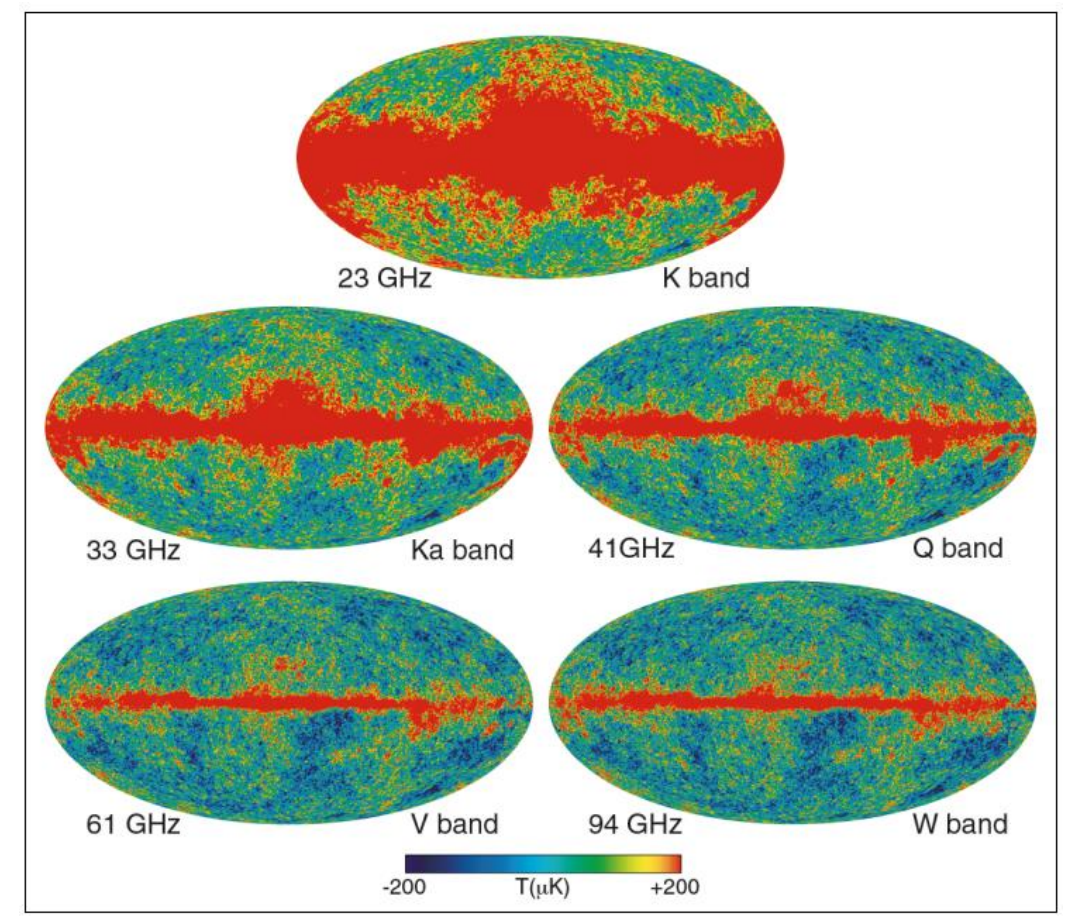

Figure 4. WMAP temperature anisotropy of the frequency spectrum of the cosmic microwave background, from [53].

The participation of correlated dichotomous pairs of $v / \mathrm{g}-\mathrm{EF}$ and the formation of a monolayer of neutrons removes the question of the baryon asymmetry of the Universe. The relic right-handed $\tilde{v}_{\mathrm{e}}$ could predetermine the sign and nature of the universal HF. Expansion of the ether space of the plasma disc to $\sim 6 \mathrm{AU}$ during the $\beta$-decay of neutrons occurred due to the scattering up and down $\tilde{v}_{\mathrm{e}}$ from the plane of the monolayer. The formation of stars and galaxies from the disk plasma could be realized according to the Alfvén-Klein model [55], and the formation of planetary systems according to the model [15]. At the same time, due to antineutrinos or EFs isomorphic to it in the ether, counterclockwise rotation prevailed above the monolayer plane, and clockwise rotation below the plane. This distribution of rotational directions is observed on spiral galaxies located above and below the Milky Way plane [56].

In this way, the implementation of the initial stage of the self-organization model of the Universe was realized in the framework of the first LD. The obedience of the Avogadro number $\left(\mathrm{N}_{\mathrm{o}}\right)$ as the coefficient of similarity of the metric parameters of the basic objects of the Universe 
testifies to the subordination of the model to the second and third laws of dialectics. In the process of $\beta$-decay of a neutron, at $\mathrm{t} \sim 900 \mathrm{~s}$, the action of an EF* with energy $\mathrm{E} \sim \mathrm{h} / \mathrm{t} \sim 710^{-30} \mathrm{erg}$ is realized. If this value is taken as the typical energy $v / g$-EF in the composition of the particles, then in the low-energy electron and low-energy neutrino their number will be $\sim 1 / 4$ from $N_{o}$, and in nucleons $\sim 500 \mathrm{~N}_{0}$. Thus, the number No is suitable as a coefficient in the following relations:

$\mathrm{m}_{\mathrm{ep}} \sim \mathrm{N}_{\mathrm{o}} \mathrm{m}_{\mathrm{EF}} ; 1 \mathrm{~g}=\mathrm{N}_{\mathrm{o}} \mathrm{m}_{\mathrm{p}}$ and $\mathrm{M}_{\supset} \sim \mathrm{N}_{\mathrm{o}} 100 \mathrm{~g}\left(\mathrm{M}_{\supset}-\right.$ mass of the Moon);

$\mathrm{R} \odot=\mathrm{N}_{\mathrm{o}} R_{H e}^{n}$ and 1 a.u. $=\mathrm{N}_{\mathrm{o}} R_{H e}^{a}\left(R_{H e}^{n}\right.$ and $R_{H e}^{a}$ radii of the nucleus and the atom of He);

$\mathrm{M}_{\mathrm{w}} \sim \mathrm{N}_{\mathrm{o}} \mathrm{M} \odot \sim 1.210^{57} \mathrm{~g}\left(\mathrm{M}_{\mathrm{w}}-\right.$ mass of the Universe $)$;

$\mathrm{N}_{\mathrm{n}} \sim \mathrm{M}_{\mathrm{w}} / \mathrm{m}_{\mathrm{n}} \sim 710^{80}\left(\mathrm{~N}_{\mathrm{n}}-\right.$ the total number of neutrons $)$;

$\mathrm{N}_{\mathrm{EF}} \sim 500 \mathrm{~N}_{\mathrm{o}} \mathrm{N}_{\mathrm{n}} \sim 210^{107} \sim N_{o}^{4.4}$ (the total number $v / \mathrm{g}-\mathrm{EF}$ ).

The fourth degree $\mathrm{N}_{\mathrm{o}}$ corresponds to four jumps in the complexity of the organization of EF in the transition from the I-th (energoform) to the V-th (Universe) level of the order of the world. With a neutron atmosphere radius of about $3 \mathrm{Fm}$ (Figure 2), the monolayer radius will be:

$$
\mathrm{R}_{\mathrm{Un}} \sim 310^{-13}\left(\mathrm{~N}_{\mathrm{n}}\right)^{1 / 2} \sim 10^{28} \mathrm{~cm} .
$$

Taking $\mathrm{R}_{\mathrm{Un}}$ for the visible horizon of the Universe, we obtain its life time of $\sim 10^{10}$ years, which is consistent with various estimates of the age of the Earth and the Universe. The value $\mathrm{R}_{\mathrm{Un}}$ itself is obtained of the same order as the Schwarzschild radius of the model of the self-closed Universe:

$$
\mathrm{R}_{\mathrm{Un}} \sim \mathrm{GM}_{\mathrm{U}} / \mathrm{C}^{2} \sim 710^{28} \mathrm{~cm},
$$

$\mathrm{G}$ is the gravitational constant. Taking into account the estimated nature of the $\mathrm{M}_{\mathrm{U}}$ values and the neutron atmosphere radius, the obtained values of the metric parameters of the Universe completely satisfy the similarity relations with the coefficient equal to $\mathrm{N}_{\mathrm{o}}$. Thus, the number $\mathrm{N}_{\mathrm{o}}$ fits the role of a bootstrap quantum that regulates quantitative and qualitative transitions between adjacent levels of the structural hierarchy of the Universe.

\section{Hypothesis about the neutrino nature of the chiral factor}

The existence of relic $\tilde{v}_{\mathrm{e}}$ and $v_{\mathrm{e}}$ has not yet been confirmed experimentally. Neutrino telescopes registered only 24 neutrinos from a supernova explosion in 1987 - 11 (Kamiokande II), 8 (IMB) and 5 pieces (Baksan) [31, 57]. To these we can add 38 ultrahigh-energy neutrinos recorded for the period 2014-2017 by the IceCube neutrino telescope [58]. Geoneutrino ( $\left.\tilde{v}_{\mathrm{e}}\right)$ occurs mainly from $\beta$-decay of ${ }^{40} \mathrm{~K},{ }^{232} \mathrm{Th}$ and ${ }^{238} \mathrm{U}$. Its registration using the reverse $\beta$-decay reaction

$$
\tilde{v}_{\mathrm{e}}+\mathrm{p}=\mathrm{n}+\mathrm{e}^{+}
$$


is complicated by the high background of the reactor antineutrinos. The threshold of this reaction is $1.8 \mathrm{MeV}$, therefore, at the Borexino and KamLAND detectors with an error of $\sim 40 \%$ and $27 \%$, they determined $\tilde{v}_{\mathrm{e}}$ from only ${ }^{232} \mathrm{Th}$ and ${ }^{238} \mathrm{U}$ [59]. The energy of $\tilde{v}_{\mathrm{e}}$ from ${ }^{40} \mathrm{~K}$ did not exceed 1.3 $\mathrm{MeV}$ and its detectors did not register.

The number of solar $v_{\mathrm{e}}$ recorded experimentally is substantially less than the Standard Model of the Sun requires. To eliminate this discrepancy, the theory of neutrino oscillations is used, which is based on the unproven assumption that the solar ve has a non-zero mass. Currently, large volumes of such substances are used as detectors: ice (IceCube); $\mathrm{H}_{2} \mathrm{O}$ (Kamiokande, Baikal); $\mathrm{D}_{2} \mathrm{O}$ (SNO); liquid $\mathrm{Ga}, \mathrm{GaCl}_{2}$ (SAGE, GALLEX); $\mathrm{C}_{2} \mathrm{Cl}_{4}$ (R. Davis); n-terphenyl + diphenyloxazole (DFO) + 1,4-di- [2- (5-phenyloxazolyl)] - benzene in white spirit (BAKSAN); dimethyloxazole in pseudocumene (Borexino); DFO in a mixture of pseudocumene + dodecane (KamLAND). The capture reactions of $v_{\mathrm{e}}$ by $\mathrm{Cl}$ and $\mathrm{Ga}$ nuclei have large thresholds and produce radioactive isotopes $\mathrm{Ar}$ and $\mathrm{Ge}$, the method of identification of which is not completely unambiguous [60]. High-energy neutrinos do not chemically interact with water, alkanes, aromatic and heterocyclic organic substances, but only ionize atoms or are scattered on nuclei and electrons. Substances and media of the detectors have the entire set of atoms $(\mathrm{H}, \mathrm{C}, \mathrm{O}, \mathrm{N})$ included in the substrate for the reaction of the prebiotic synthesis of chiral amines and sugars.

Considering this and the negligible probability of interaction of $v_{\mathrm{e}}$ with telescope detectors, it can be concluded that cosmogenic and solar neutrinos cannot cause chiral imbalances in the synthesis of amines and sugars at the chemical and prebiotic stages of evolution [8, 61]. The solar $v_{\mathrm{e}}$ does not also interact with radioactive nuclei, as indicated by the absence of seasonal variations for the reaction rates of $\alpha$ - and $\beta$-decay of radioactive elements $[62,63]$, while annual variations are observed for the solar neutrino of the beryllium cycle [64, 65]. Obtaining reliable data on variations in the flux of the solar neutrino day/night is very difficult due to the significant difference between the night and daytime radiation backgrounds [66].

Regarding geoneutrino, it is believed [61] that the content of the main sources of $\tilde{v}_{\mathrm{e}}$ in the earth's crust $\left({ }^{232} \mathrm{Th},{ }^{238} \mathrm{U}\right.$ and $\left.{ }^{40} \mathrm{~K}\right)$ during the Archean era was more than now at $\sim 1.5 ; \sim 2$ and 20 times, respectively. Thus, the terrestrial $\tilde{v}_{\mathrm{e}}$ could, in principle, play the role of CF in the genesis of homochirality in the Archean era, however, as in the case of solar ve, this requires an adequate mechanism for the asymmetric effect of neutrinos on the chemical synthesis of simple chiral 
molecules, and then on the processes of formation and functioning of biological molecules in living systems.

In $[35,36]$, taking into account the negligible probability of interaction of $\tilde{v}_{\mathrm{e}}$ and $v_{\mathrm{e}}$ with matter, the incompleteness and purely statistical nature of the experimental data on neutrinos [60, 67,68 ], by analogy with the dissipation of hydrodynamic vortices [69], it was assumed that $\tilde{v}_{\mathrm{e}}$ and $v_{\mathrm{e}}$ low energies in vacuum decay into $\mathrm{N}_{\mathrm{o}}$ energoforms ( $v$-decay) isomorphic to their structure $\left(v_{\mathrm{e}^{-}}\right.$ EF). After $v$-decay, $v_{\mathrm{e}}$-EFs can be embedded in the electric and magnetic field lines [70, 71] and combined with photons to form hybrid photon-neutrino EF ( $v_{\gamma}$-EF) [72].

ve-ЭФ, from which unstable structures $\tilde{v}_{\mathrm{e}}$ and $v_{\mathrm{e}}$ are formed are superfluous in energy and incompatible by symmetry for the structures of nucleons and electrons. The EF* responsible for the $\beta$-decay of a neutron, apparently, is captured by the $\tilde{v}_{\mathrm{e}}$ structure and determines its $v$-decay for a time $t\left(E_{v} / E_{n}\right) \sim 1 s$, where $E_{v}$ and $E_{n}$ are the energies of $\tilde{v}_{e}$ and the neutron, respectively. The fast $v$-decay of low-energy solar $v_{e}$ pp-type [73] is due to the different directions of the spins of its shell and orbitals (Figure 3). Non-accelerator physics of elementary particles allows neutrino instability [74], but $v$-decay is not taken into account by high-energy physics, since its objects are mainly high-energy neutrinos $[51,75]$. Their lifetime, structure and nature of the interaction with the substance can vary significantly due to the effect of encapsulation.

The hypothesis of $v$-decay and the neutrino nature of CF suggests the possibility of the absorption of $v_{\mathrm{e}}$-EF by living matter in the process of interaction with the magnetic moments of the nuclei, protons and electrons of the molecules forming the structurally ordered and dynamic correlated system [72, 76, 77, 78]. Such systems consist of water and homogeneous chiral molecules or hormone-enzyme-substrate type complexes. Water of dynamic clusters and hydrated shells plays the role of a matrix and a proton conductor, limiting thermodynamics and physicochemical properties of biosystems $[3,7,79,80,81]$. An increase in the level of spinmagnetic coherence of the system during its absorption of $v_{\mathrm{e}}$-EF leads to an increase in the degree of its orderliness and chiral complementarity of the metabolites. Accordingly, the barriers of dark chemical reactions initiated by tunneling electron or proton transitions decrease, and the chemical potential of the biosystem will eventually increase [8].

The dependence of specific rotation $[\alpha]$ (optical activity) of polysaccharide saline (dextran) on $\mathrm{T}$ and its seasonal monitoring show the possibility of $v_{\mathrm{e}}-\mathrm{EF}$ absorption by chiral biosystems and participation of water in this process and its seasonal monitoring is presented in Figure 5. The 
estimated activation energy $\left(E_{A}\right)$ from the Arrhenius approximation, the dependence of $[\alpha]$ on $T$ for a dextran solution gave a value of $0.38 \mathrm{~kJ} / \mathrm{mol}$, close to $\mathrm{E}_{\mathrm{A}}$ for physiological glucose solution $-0.33 \mathrm{~kJ} / \mathrm{mol}[81]$ and comparable to the value of excitation energy of the first rotational state ortoizomera $\mathrm{H}_{2} \mathrm{O}(0.28 \mathrm{~kJ} / \mathrm{mol})$ [82]. The increase in the value of $[\alpha]$ dextran solution after March 22 (Figure 5b) correlates with the process of spring awakening of plants and the annual variation of the solar $v_{\mathrm{e}}[64,65]$. The biogenicity of the terrestrial $\tilde{v}_{\mathrm{e}}$ confirms the correlation of the anomalously high growth rate of coniferous trees in the Tungus catastrophe region with the kinetics of $\beta$-decay of radioactive elements trapped in the soil after a meteorite explosion. These results confirm the hypothesis about the neutrino nature of $\mathrm{CF}(\mathrm{vCF})$ and its participation in the metabolism of living organisms.

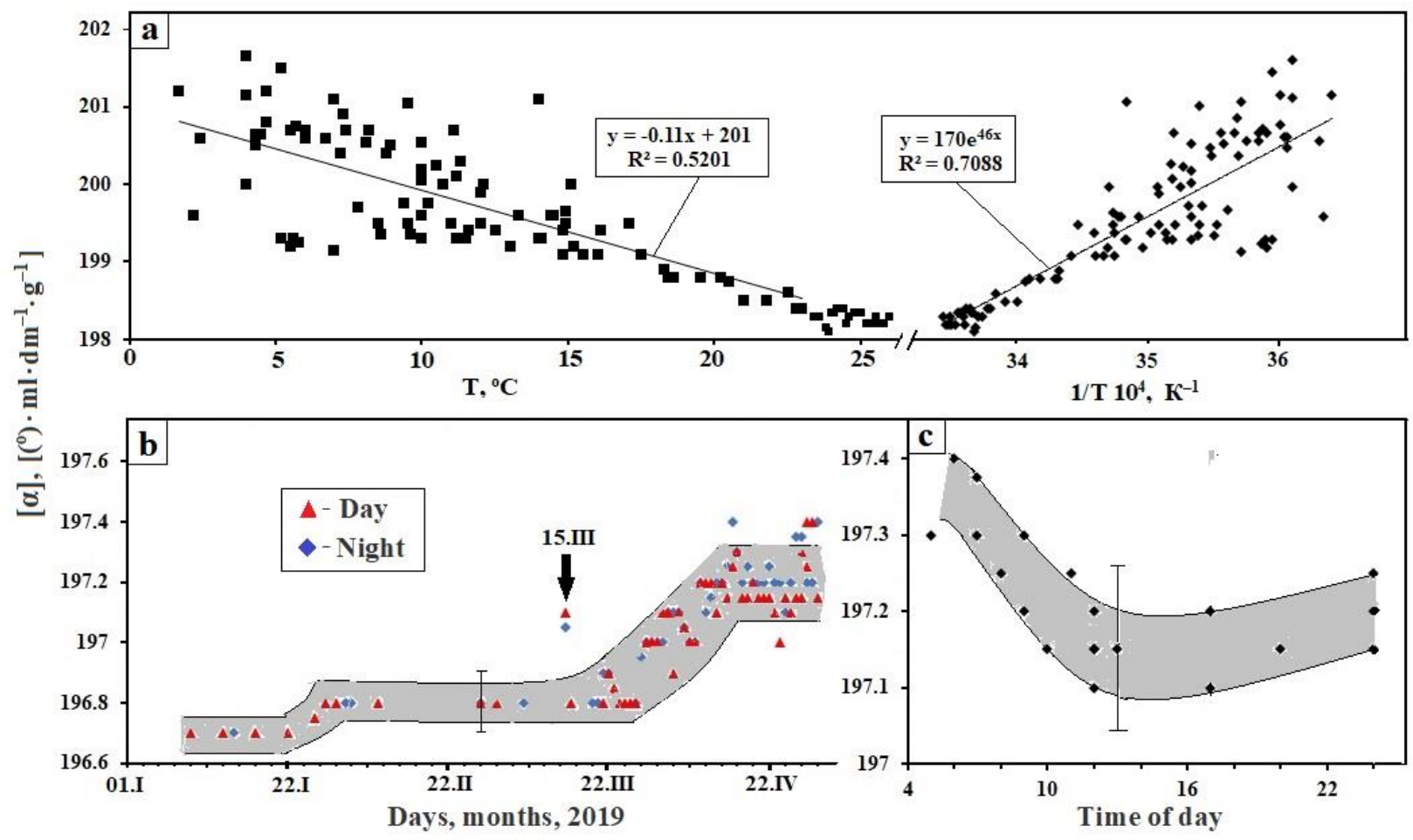

Figure 5. (a) Dependence of specific rotation [ $\alpha$ ] of dextran saline on $T$ and $1 / T$ (Arrhenius approximation), from [81]. (b) Dependence [ $\alpha$ ] in the period from 04. I.2019 - 30.IV.2019, the arrow indicates the values [ $\alpha]$ with the index of magnetic activity $K p=6$ (15.III.2019). (c) Daily variations $[\alpha]$ in the period 10.IV-01.V. 2019. Toned areas of fluctuations of the measured values [a] [81].

The participation of $v \mathrm{CF}$ in the genesis of homochirality at the prebiotic (chemical) stage of evolution could have taken place in the Archean era $\sim 4$ billion years ago with the dim Sun. Under the conditions of the warm and humid climate of the Archaean [83, 84], vCF could modulate the kinetics of thermo- and electrochemical fusion reactions in the initial substrate. The high 
density of the right-handed $\tilde{v} e$ in the Archean era determined the dominance of the catalytic synthesis of D-sugars $[28,85]$. After the terrestrial sources of $\tilde{v}_{\mathrm{e}}$ burnout, the predominance of the left-handed solar $v_{\mathrm{e}}$ provided the synthesis of L-amines and in further evolution could play a role vCF [8]. The chiral differentiation of the synthesis of sugars and amines, and then of complex molecules (proteins, proteids, enzymes), could be due to the predominance of oxygen in the structures of sugars and the presence of $\mathrm{N}$ in amines. The nuclear spin of nitrogen in amino acids, and then proteins, could serve as an important element of the gene code sensitive to $\mathrm{VCF}$ [78]. At the same time, the right D-sugars turned out to be sources of right handed quantum of metabolic EM energy. As a result, already in the Archean era, archebionts arose and then communities from anaerobic prokaryotes.

With the release of solar energy to the proterozoic solar energies in the biosphere, the role of photochemistry producing oxygen and ozone increased, and cyanobacterial communities and photosynthesizing prokaryotes dominating in them began to develop rapidly. In this case, the decay products of anaerobic prokaryotes served as chiral substrates. The further evolution of the biosphere took place under the conditions of energy-information synergism of solar photons and solar neutrinos, operating mainly at night (Figure 5c).

\section{Physical and chemical features of chirality in biology}

If the importance of the problem of neutrinos has been realized in physics of EP and cosmology and many experimental and theoretical studies are devoted to its solution, the biogenicity of neutrinos and $v$-EF does not figure in any way in modern sciences. Meanwhile, it is possible that it is precisely the knowledge of the role of $v \mathrm{CF}$ in biophysics that will open the physical nature of the neutrino, and with it the window to the new physics [86]. A promising direction of studying the mechanism of action of $\mathrm{VCF}$ in the biosphere and noosphere will be to establish the role of the chiral physicochemical component in phenomena that have not yet received a reasonable scientific explanation $[87,88]$. The following can be attributed to such phenomena.

1). Cold stratification of plant seeds [76, 89, 90];

2). Gigantism among fishes (nanism) dwelling at the great depths of Baikal [91];

3). The predominance of the right shells in mollusks and human sweat ducts [7,92];

4). Walking people in a circle in the desert and the forest [93] and rotating the body while running in place [31]; 
5). Generation of the functional asymmetry of the brain in a dream [7, 31, 41];

6). The maximum content of cortisol and melatonin in the blood at night and in the early morning [76, 94, 95, 96];

7). Seasonal variations of the physical and mental state of a person [94, 96];

The anomalous nature of most of the above phenomena, in principle, can be explained by the action of the $v_{\mathrm{e}}$-EF flow coming out of the earth at night when the EM factor of the Sun is absent and the metabolism of animals and seeds of plants in the state of sleep and rest significantly changes. Anomalous phenomenon 1) is that keeping the swollen seeds in the dark under moist quartz-containing sand at a temperature of about $4{ }^{\circ} \mathrm{C}$ leads to the activation of enzymes and the start of seed germination [76, 89, 90]. Under natural conditions, such a cold treatment is experienced by the seeds of perennial plants during the climatic transition from winter to spring. The cereal seed endosperm is a homogeneous fine medium of polycrystalline starch grains consisting of two types of $\alpha$-D-glucose polymers - linear amylose polymer and branched amylopectin polymer (Figure 6) [76]. The anisotropy of starch structure is manifested by birefringence in polarized light. Phytohormone (+)-Abscisic acid, which has a very high chirality [76], plays an important role in the process of awakening seeds [76] and its synthesis in a plant at night is 50-60 times more efficient than during the day. The processes of awakening and seed germination are sensitive to external alternating and permanent magnetic fields [76, 90]. Phenomena 2), 3) are also observed in biosystems containing water or being in water and incorporating chiral metabolites or spiral supramolecular formations.

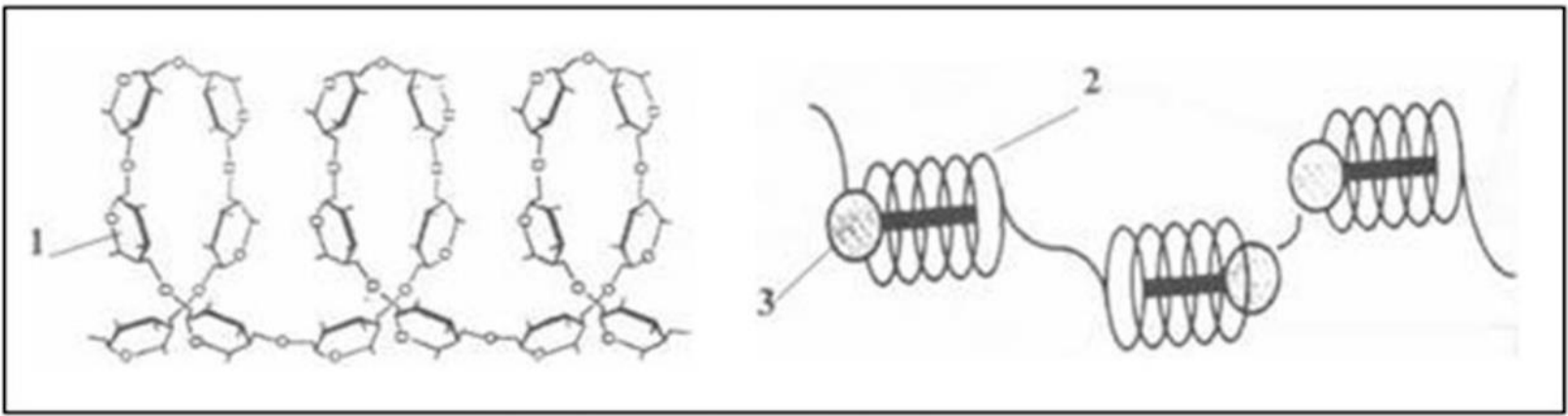

Figure 6. Elements of the structure of polymers amylose and amylopectin: glucose residue (1); amylozolipid complex - amylose helix (2); lipid (3) [76].

An essential role in the adaptation of animal physiology to the action of $v$-EF, apparently, was played by the epiphysis, which has unique physicochemical properties [96, 97, 98]. The high flux density of $v_{\mathrm{e}} \mathrm{EF}$ in the surface layer and in water bodies caused the development of a 
subcutaneous third (parietal) eye in reptiles that has a neural connection with the epiphysis, and in deep-water fish a pigment spot formed above the epiphysis [97]. The latter fact indicates the possibility of participation of $v_{\mathrm{e}}$ EF in phenomenon 2). This was facilitated by a high degree of clustering of the water of Baikal at its average annual temperature at a depth of $4{ }^{\circ} \mathrm{C}$ [3]. Since the lake bed is formed by a fracture of the Earth's crust, reaching the upper layers of the mantle, at its depths the flux density emanating from the earth, $v_{\mathrm{e}}-\mathrm{EF}$, can be increased. The third eye of reptiles has the same elements as ordinary eyes, but is not designed for the direct action of light.

In an adult, $v_{\mathrm{e}}$-EF and $v_{\gamma}$-EF can condense in the subcutaneous tissue containing up to $75 \%$ water and a high concentration of hyaluronic acid polysaccharide. The role of the condenser and lenses for $v_{\mathrm{e}}-\mathrm{EF}$ and $v_{\gamma}$-EF, obviously, is played by the vitreous body of the eyes and the venous sinus, located under the fontanel [41]. The following facts indicate the possibility of the participation of chiral EFs emanating from the earth in human embryogenesis. In the division of the egg and the subsequent development of the embryo in the process of transferring genetic information from inducer molecules to the embryo, $v_{\gamma}$-EF can participate through the mediation of fluids bordering the embryo disk [99]. The participation of $v$-EF in brain development is evidenced, for example, by head previa of the fetus in the third trimester of pregnancy and openness of the fontanel before the age of two. In principle, the distribution of $v_{\gamma}$-EF in biotissues must obey the laws of wave optics [100]. This, for example, is indicated by the concentric distribution of cones in the neuroretin with a maximum in the center of the fovea [101]. Such a distribution is similar to the Fraunhofer diffraction pattern and can be explained by the diffraction of the $v_{\gamma}$-EF flux on the slit of the future pupil of the eye.

The whole complex of morphofunctional features of the epiphysis of animals and humans is consistent with the assumption of the participation of ve-EF in its phylogenesis [41]. In humans, the epiphysis has become the center responsible for the circadian rhythm and regulating the physiology of virtually all vital organs, mainly through the neurohormone melatonin (ME), synthesized in the epiphysis. The preservation in the epiphysis of the mechanism of sensitivity to the action of $v_{\mathrm{e}}$-EF can be judged by the increase in its biochemical activity not at midnight (middle of the night), but at 2-4 hours. It was at this time that the maximum ME content in venous blood and cerebrospinal fluid is observed, with the ME in the lateral ventricles 7 times more than in the blood and 20 times in the third ventricle [94]. And the middle of sleep, which lasts for 8 hours, also falls on 2-3 hours of the night. Acceleration of ME biosynthesis at 2-4 nights can be associated 
with the night maximum flux density $v_{\mathrm{e}}$-EF (Figure 5c) and their accumulation efficiency in pinealocytes and epiphysis parenchyma due to minimization of body temperature (Figure 7).

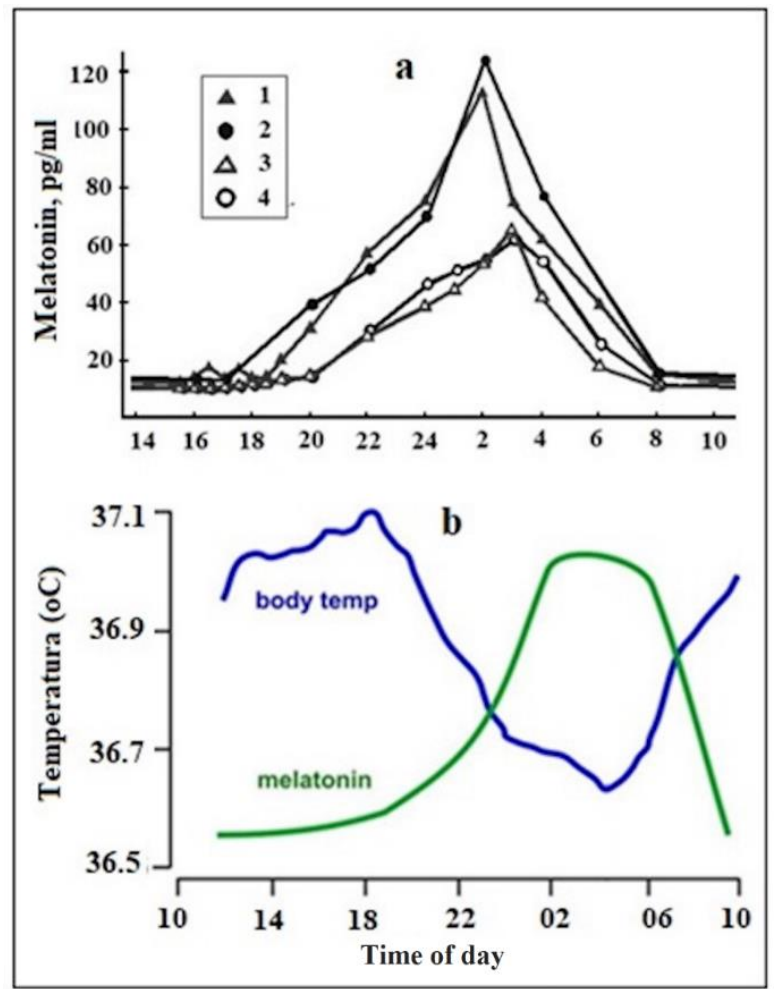

Figure 7. (a) the content of melatonin in blood plasma at different times of the day: 1, 2 boys and girls; 3, 4 - elderly men and women, from [95]. (b) the daily temperature of the human body and the content of melatonin in the blood plasma, from [102].

Chiral L-norepinephrine and L-tryptophan are involved in the biosynthesis of ME, and its kinetics is limited by the enzyme arylalkylamine-N-acetyltransferase, whose activity depends on the person's age (Figure 7) and increases 100 times at night [94]. The synthesis of ME is influenced by an alternating magnetic field, both natural and technogenic [103]. The ganglion neurons of the retina and the neurons of the suprachiasmatic nuclei have spontaneous, rhythmic activity in the dark [41, 104]. The molecular structure of the brain sand crystals of the human pineal gland in addition to the piezoelectric effect in polarized light reveals birefringence in the form of a "Maltese cross" [105]. The sand content in the epiphysis of schizophrenics is significantly below the norm [106], while in the prodigy the epiphysis was abnormally large [97].

The genesis and activation of phenomena 4) - 7) in [31] was associated with the action of $v_{\mathrm{e}}$-EF on the back side of the Earth. The $v_{\mathrm{e}}$ EF flow flows around the Earth through a layer of lithosphere with a thickness of $\Delta \mathrm{R}$, extending to the boundary of Makhorovichich (Figure 8). This 
layer consists of $\sim 70 \%$ silica containing equal amounts of L- and D-crystals of quartz [8]. On the night side of the Earth, the oncoming $v_{\mathrm{e}}-\mathrm{EF}$ flows merge and come to the surface. The shift of the maximum rate of ME synthesis in the epiphysis from midnight at 2-4 o'clock in the morning was associated with the achievement at this time of the maximum density of the $v_{\mathrm{e}}$-EF flux coming out of the earth. The time shift was explained by the rotation of the Earth $(\omega)$ and estimated the velocity of the $v_{\mathrm{e}}$-EF flow in the lithosphere as a waveguide (Figure 8).

Taking into account the speed of rotation of the Earth $\left(V_{E}=\omega R_{E}\right)$ and the inclination of its axis of rotation $\left(23^{\circ}\right)$, it was possible to draw up an equation for the time of movement $(t)$ of the flows $v_{\mathrm{e}}-\mathrm{EF}$ to the point of their merging:

$$
t=\frac{\pi R_{\mathrm{E}}-\frac{1}{6} \pi R_{\mathrm{E}}}{V_{v}-V_{\mathrm{E}} \cos 23^{0}}=\frac{\pi R_{\mathrm{E}}+\frac{1}{6} \pi R_{\mathrm{E}}}{V_{v}+V_{\mathrm{E}} \cos 23^{\circ}}
$$

the solution of which gave $\mathrm{V}_{v} \sim 2.8 \mathrm{~km} / \mathrm{s}$. Knowing $\mathrm{V}_{v}$ and $\omega$, we calculated the displacement of the exit point $v / g-E F$ in longitude $(S)$ relative to the diametral point of entry of the flow $v_{\mathrm{e}}$-EF into the ground:

$$
S \sim t V_{\mathrm{E}} \sin 23^{\circ} \sim 0.07 \pi R_{\mathrm{E}} \sim 1400 \mathrm{~km} \text { or } \sim 13^{\circ} .
$$

The estimated $\mathrm{V}_{v}$ flux $v / \mathrm{g}$-EF in the lithosphere agrees well with the velocity of surface seismic waves in the Earth's crust (3.0-4.4 km/s [107]) modeled by quasi-particles (magnon and polaron). The dynamics of these perturbations of the lithosphere can correspond to the mechanism of movement along it $v_{\mathrm{e}}$-EF. The value of $\mathrm{V}_{v}$ varies depending on the coordinates of the entrance of $v_{\mathrm{e}}$-EF flows and seasonal changes in the orientation of the axis of rotation of the Earth relative to the Sun.

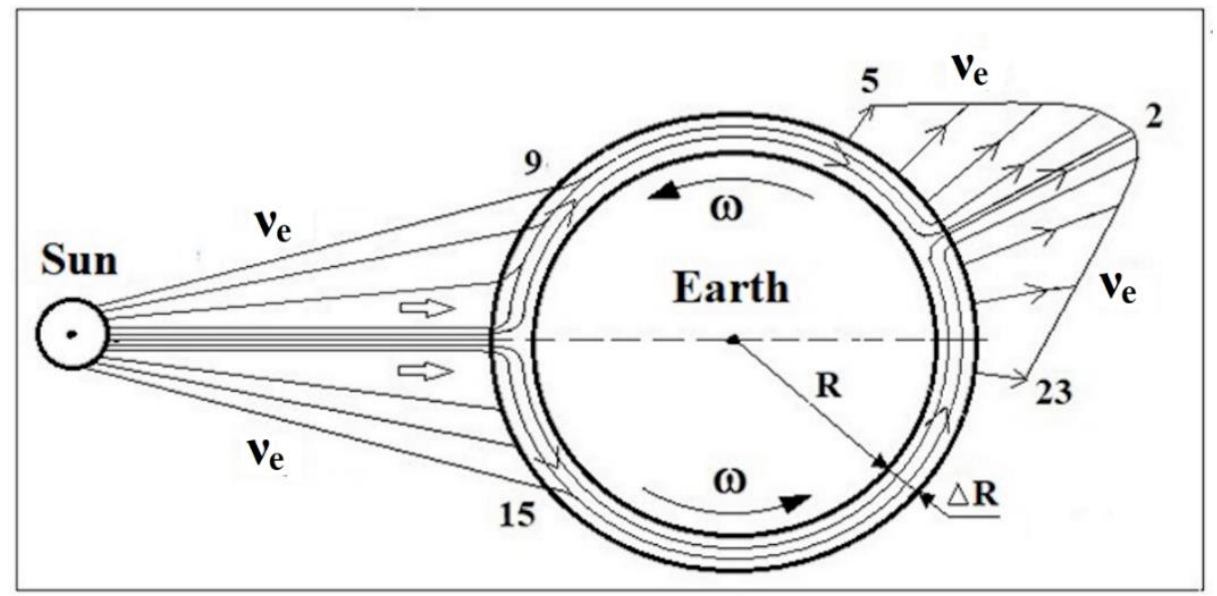

Figure 8. Scheme of the flow of neutrino energoforms $\left(v_{e}\right)$ in the layer of the lithosphere with a thickness of $\Delta R \sim 10-50 \mathrm{~km}$; the numbers are the time of day in hours, from [96]. 
Seasonal variations in the $v_{\mathrm{e}}$-EF flux and its dependence on solar activity can be judged by the increase in $[\alpha]$ dextran saline during the transition from winter to spring and by the jump $[\alpha]$ March 15, 2019 (Figure 5b), as well as a decrease in biosynthesis efficiency ME in the epiphysis in winter [94]. Regular homeostasis reactions to a decrease in ME concentration in the blood will increase the volume of the parenchyma and the number of pinealocytes, which is consistent with an increase in the size and mass of the epiphysis in people who lived in the middle latitudes of the Northern Hemisphere and died in November-January compared to people of similar constitution who died in May-June [94].

In the science of the brain, it remains an open question about the dependence of its functional asymmetry on the distribution of the sign of the spirals of the myelin sheaths of nerve fibers in the hemispheres of the brain. Taking into account the presence in the layers of myelin spiral channel (notches), filled with cytoplasm, the myelin segment of the nerve fiber was presented by an inductor [41, 96, 104] (Figure 9). The action potential in the interception of Ranvier is generated by increasing the concentration of $\mathrm{Na}^{+}$in the axoplasm of the paranoid region of the myelin sheath. In this case, a bias current is excited, the front of which moves along notches spirals and simultaneously along the axon of the axon. According to the law of EM induction, the bias current generates a vortex magnetic field, the front of which will move along the axon at a speed of V $\mathrm{C} / \mathrm{n}$ ( $\mathrm{n}$ is the refractive index of axoplasm). Thus, the speed of the saltatory conduction of myelin fiber will limit the kinetics of the processes in the interception of Ranvier. The sign of the myelin helix determines the direction of the bias current and the vector B in accordance with the rule of the right screw (Figure 9). The geometry of the EM vortices is, in principle, similar to the $v / \mathrm{g}$-EF (Figure 1), and their EM nature is related to biophotons and IR photons appearing in [100, 108, 109, 110].

The equivalent circuit scheme of the nerve membrane contains a resistance and a capacitor, with the introduction of an inductance element, an oscillating circuit is obtained. The possibilities of modeling the properties of the neuron and the brain are greatly expanded, and they acquire the property of chirality. The photon-like $v_{\gamma}$-EFs generated in the neural networks of each hemisphere form a single quantum system. Changes in its state and the interaction between chiral EM quanta formally obey the Bose-Einstein distribution and transitions (1). 


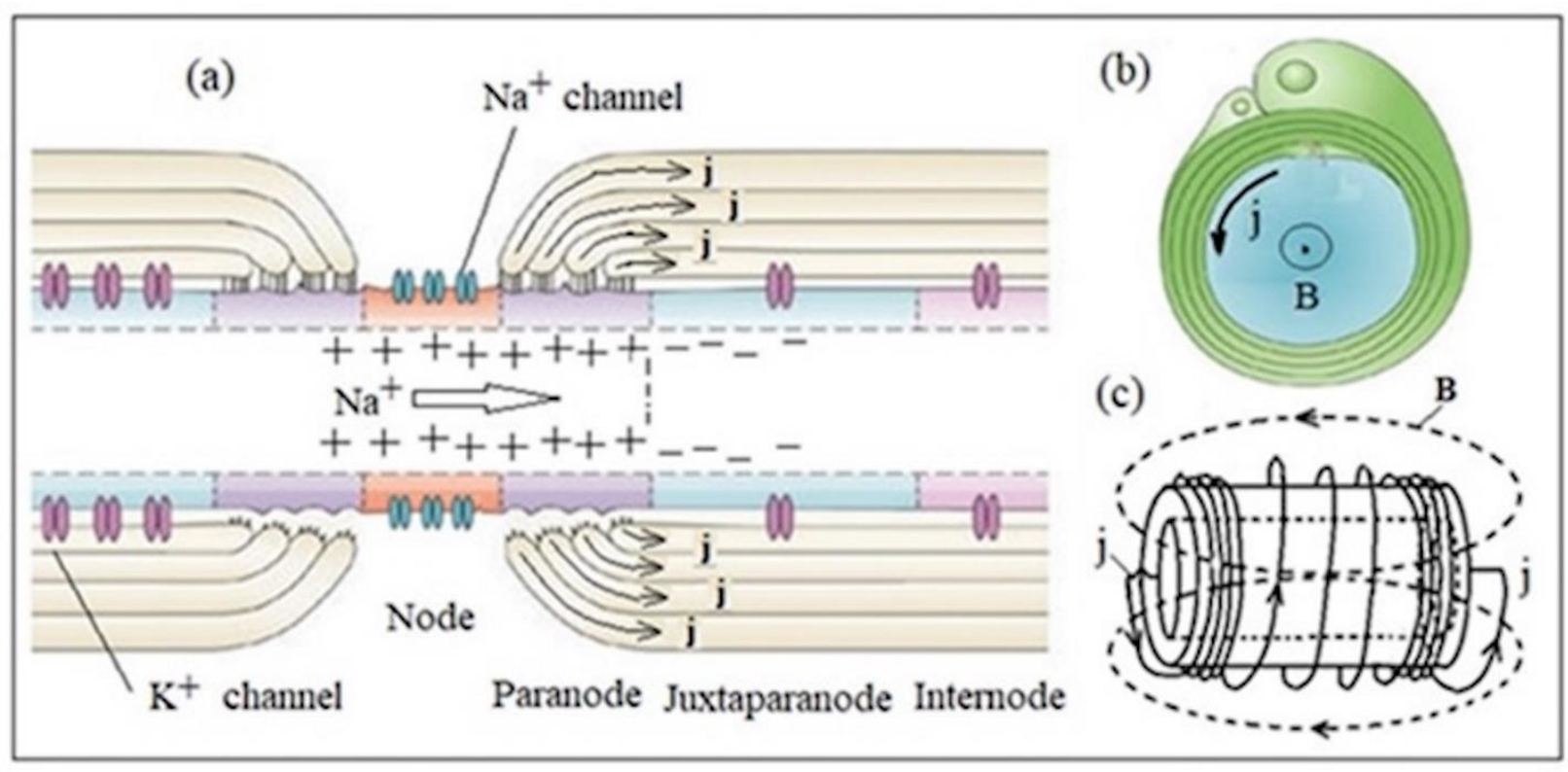

Figure 9. Induction model of the soltator mechanism of nerve impulse transmission. Scheme of interception of Ranvier (a), myelin sheath $(b)$ and myelin nerve segment $(c)$. The arrow shows the diffusion of $\mathrm{Na}^{+}, \boldsymbol{j}$ - the displacement currents, $B$ - the vortex magnetic field, from [104].

The whole brain can be modeled by a two-module processor; its multi-level hierarchy of synaptic connections in the cerebral cortex will serve as its elemental base. The paired structures of the subcortex, together with the cerebrospinal fluid system, the thalamus and the epiphysis, will play in the model the role of commuting, signaling and control elements, responsive to internal and external signals, taking into account their chirality.

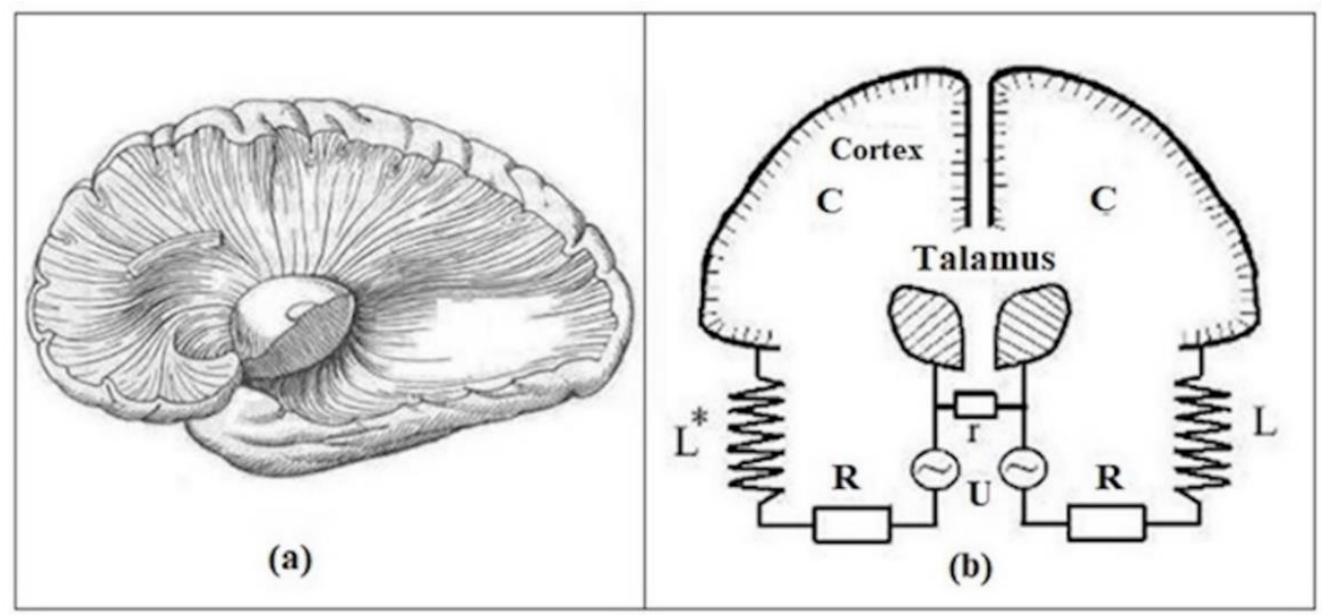

Figure 10. (a) Equivalent oscillatory circuits that simulate the brain's electrophysics. (b) $L^{*}, L, R$ - inductive and ohmic resistances of subcortical structures of the right and left hemispheres; $r$ is intertalamic fusion; $C$ and $U$ - capacity and potential difference between the thalamus and the cortex, from [41]. 
The baseline $\alpha$-, $\mu$-rhythms [104] and functional rhythms of the brain-processor are determined by the frequencies of the oscillatory circuits formed from subcortical structures and areas of the cortex that are involved in the implementation of the corresponding brain function. The electrophysics of the thalamus plays a key role in the functional differentiation of the complex hierarchy of oscillatory circuits, which reflects the integral equivalent electrical circuit of the brain (Figure 10).

\section{Conclusion}

The review revealed the fundamental nature of the problem of the origin of the homochirality of living systems. To solve it, the energoforms paradigm was proposed and substantiated, the construction of which was guided by the recommendation of Werner Heisenberg: only those who try to change as little as possible can expect to succeed, showing that the changes are forced by the subject itself, and those small changes that the absolute necessity is finally proved, then forced to change the structure of thinking, that is, to produce fundamental changes [1]. The logic of the paradigm was based on the laws of dialectics, electromagnetism and the principles of certainty of quantum of action at the level of energoforms. In the framework of the paradigm of energoforms, we created a physically adequate algorithm for modeling chiral energoforms, structures of elementary particles and nuclei in the ground and excited states. The algorithm was successfully tested by calculating and systematizing the dynamic and metric characteristics of the main particles and more than one hundred nuclei. In the calculation, reliable empirical parameters of particles and nuclei were used as boundary conditions. As a solution to the problem of the neutrino and the physical nature of the universal chiral factor, a hypothesis was put forward regarding the instability of the neutrino and the biogenicity of its decay products chiral energoforms. In confirmation of the hypothesis, the physicochemical features of the chiral phenomena of nature were associated with possible mechanisms for the participation of chiral energoforms in them. Using the paradigm of energoforms, we built a hierarchy of the chiral dichotomy of the world and defined it as the highest level of complexity, the chirality of the physiology of the heuristic thinking of man. 
25

\section{Literature}

1. Giudice, G. F. The Dawn of the Post-Naturalness Era, https://arxiv.org/abs/1710.07663v1

2. Heisenberg, V. Changes in the structure of thinking in the development of science. Steps beyond horizon. M., Russia, 1987, pp. 190-200

3. Kholmanskiy, A.; Zaytseva, N. Physically adequate approximations for abnormal temperature dependences of water characteristics. J. Mol. Liq. 2019, 275, pp. 741-748.

https://doi.org/10.1016/j.molliq.2018.11.059

4. Ostrovskii, V.E.; Kadyshevich, E.A. Thermodynamics of formation of nitrogen bases and Dribose from mineral substances in light of the problem of origination of simplest elements of living matter. Thermochim. Acta 2006, 441, pp. 69-78

5. Rubakov, V. The emergence of new physics is inevitable. Dark Energy, Dark matter 2015, URL: http://victorpetrov.ru/valerij-rubakov-poyavlenie-novoj-fiziki-neizbezhno.html

6. Blumenfeld, L.A. Problems of biological physics. Science, M, Russia, 1977, 336 p.

7. Kholmanskiy, A.S. Chirality and quantum effects as factors of morphogenesis. Electron.

Math. Med.-Biol. J. 2010. 9(4) URL: http://www.sci.rostelecom67.ru/user/sgma/MMORPH/N28-html/kholmanskiy-2/kholmanskiy-2.htm

8. Kizel', V. A. Physical Causes of Dissymmetry of Living Systems, M, Russia, 1985, 120 p.

9. Goldanskii, V.I. Kuz'min, V.V. Spontaneous Breaking of mirror symmetry in nature and the origins of life. Sov. Phys. Usp. 1989, 32, pp. 1-29.

10. Blackmond, D. G. The origin of biological homochirality. Phil. Trans. R. Soc. B, 2011, 366, pp. 2878-2884. doi:10.1098/rstb.2011.0130.

11. Brazil, R. The origin of homochirality. Chemistry World, 2018, URL:

https://www.chemistryworld.com/feature/the-origin-of-homochirality/9073.article

12. Tsarev, V. A. Physical and astrophysical aspects of the problem of origin of chiral asymmetry of the biosphere. Phys. Particles and Nuclei, 2009, 40(7), pp. 998-1029.

13. Meierhenrich U. Amino Acids and the Asymmetry of Life: Caught in the Act of Formation. Berlin etc.: Springer, 2008. 241 p.

14. Cline, D.B. Possible Physical Mechanisms in the Galaxy to Cause Homochiral Biomaterials for Life. Symmetry, 2010, 2, pp. 1450-1460; doi:10.3390/sym2031450.

15. Snytnikov V. N., Parmon V. N. Life creates the world. Science First Hand. 2004. 1. pp. 2031) URL: https://scfh.ru/papers/zhizn-sozdaet-planety/ . 
16. Cline, D.B. Supernova antineutrino interactions cause chiral symmetry breaking and possibly homochiral biomaterials for life. Mendeleev Commun. 2004, 14, pp. 301-304.

17. Bargueño, P.; Gonzalo, I. Effect of cosmological neutrinos on discrimination between the two enantiomers of a chiral molecule. Origins Life Evol. B. 2006, 36, pp. 171-176.

DOI: $10.1007 / \mathrm{s} 11084-005-9005-2$

18. Bargueño, P.; Tudela, R.P. The Role of Supernova Neutrinos on Molecular Homochirality. Orig Life Evol Biosph, 2007, 37, pp. 253-257, DOI 10.1007/s11084-006-9060-3

19. Boyd, R.N.; Kajino, T.; Onaka, T. Supernovae, neutrinos, and the chirality of the amino acids. Int. J. Mol. Sci. 2011, 12, 3432-3444.

20. Famiano, M. Boyd, R. Kajino, T. et al. Determining Amino Acid Chirality in the Supernova Neutrino Processing Model, Symmetry 2014, 6(4), 909-25; https://doi.org/10.3390/sym6040909.

21. Famiano, M.A. Boyd, R.N. Kajino, T. Onaka T. Selection of Amino Acid Chirality via Neutrino Interactions with ${ }^{14} \mathrm{~N}$ in Crossed Electric and Magnetic Fields. Astrobiology. 2018 18(2) Published Online: https://doi.org/10.1089/ast.2017.1686

22. Meinert C., Myrgorodska I.,de Marcellus P., Buhse T., Nahon L., Hoffmann S.V., Le Sergeant d'Hendecourt L., Meierhenrich U. J.: Ribose and related sugars from ultraviolet irradiation of interstellar ice analogs. Science, 2016, 352, pp. 208-212.

23. Meinert, C., Filippi, J.-J., Nahon, L. et al. Photochirogenesis: Photochemical models on the origin of biomolecular homochirality. Symmetry, 2010, 2, 1055-1080.

24. Tsubasa, F. T., Tamura, M., Kandori, R. et al. Extended High Circular Polarization in the Orion Massive Star Forming Region: Implications for the Origin of Homochirality in the Solar System. Orig Life Evol Biosph, 2010, DOI 10.1007/s11084-010-9206-1

25. Kwon, J.; Tamura, M.; Lucas, P.W. et al. Near-infrared circular polarization images of NGC 6334-V. Astrophys. J. Let. 2010, 765(1) doi:10.1088/2041-8205/765/1/L6

26. Myrgorodska I., Meinert C., Martins Z., d'Hendecourt L., Meierhenrich U. J.: Molecular chirality in meteorites and interstellar ices, and the chirality-experiment onboard ESA's cometary Rosetta mission. Angew. Chem. Int. Ed. 2015, 54, pp. 1402-1412

27. Saito, T., Tsarev, V. Explosion of supernew as a source of chiral exposure in the space scenario of life. Bull. Lebedev Phys. Inst. 2004, 3, p. 12. 
28. Gusev, G.A. \& Guseva, Z.G. Type 1A Supernova Explosion and the Origin of Sugar Chiral Asymmetry in Biological Systems. Ibid. 2018. 45: p. 145. DOI:

$\underline{10.3103 / \mathrm{S} 1068335618050044}$

29. Dreiling, J. M.; Gay, T. J. Chirally Sensitive Electron-Induced Molecular Breakup and the Vester-Ulbricht Hypothesis. Phys. Rev. Lett. 2014, 113, 118103, https://doi.org/10.1103/PhysRevLett.113.118103

30. IceCube Collaboration, Neutrino emission from the direction of the blazar TXS 0506+056 prior to the IceCube-170922A alert. Science, 2018, 361 (6398) pp. 147-151; DOI:

10.1126/science.aat2890; URL: http://science.sciencemag.org/content/361/6398/147

31. Kholmanskiy, A.S. Dependence of the resource of functional asymmetry of the brain on external conditions. The galactic factor of spiritual evolution. Asymmetry. 2009, 3 (1), pp. 51-71.

URL: http://cerebral-asymmetry.narod.ru/Asymmetry_1_2009.pdf

32. Kawai, J. Comment on "Ribose and related sugars from ultraviolet irradiation of interstellar ice analogs", Technical Report in Science. 2017. 355(6321) 2017.

DOI: $\underline{10.1126 / \text { science.aag2990. }}$

33. Adorno, T.V. Negative dialectic. Scientific world, M, Russia, 2003, 374 p.

34. Maxwell, J. Selected Works on the Theory of the Electromagnetic Field. M. 1954, 688 p.

35. Kholmansky, A. Beginning of theophysics. M. Russia, 1999. 124 p. ISBN 5-86020-375-6.

URL: https://dlib.rsl.ru/viewer/01000633036\#?page=1

36. Kholmanskiy, A. Fractal-resonance principle of action. 2003, MIS-RT, 29(2). URL: http://www.ikar.udm.ru/sb/sb29-2.htm .

37. Kholmanskiy, A. Elementary physics of the ether, Science and World. Int. Sci. J. 2016, 1(4).

pp. 19-24, URL: http://scienceph.ru/d/413259/d/science_and_world_no_4_(32),_april,_vol._i.pdf

38. Kholmanskiy, A.S. Classification and parameters of excited states of elementary particles,

Ibid. 2016, 1(5). pp. 30-35. URL:

http://scienceph.ru/d/413259/d/science_and_world_no_5_33_may_vol_i.pdf

39. Kholmanskiy, A.S. Structure of nucleus and periodic law of Mendeleev. Electron. Math.

Med.-Biol. J. 2017, 16(1). URL: http://new-idea.kulichki.net/pubfiles/180419003144.pdf

40. Kholmanskiy, A.S. Electromagnetic nature of relativistic effects. Ibid, 2008, 7(3) URL:

http://sgma.alpha-design.ru/MMORPH/N-19-html/kholmanskiy-2/khomanskiy-2l.htm 
41. Kholmanskiy, A. Modeling of brain physics. Ibid, 2006, 5 (4). URL; http://newidea.kulichki.net/pubfiles/180520162644.pdf.

42. De Broglie, L. et al, Rotator Model of Elementary Particles Considered as Relativistic Extended Structures in Minkowski Space. Phys. Rev. 1963. 129(1) pp. 438-450.

DOI: $10.1103 /$ PhysRev.129.438

43. Ginzburg, V. L. On superconductivity and superfluidity (what I have and have not managed to do), as well as on the 'physical minimum' at the beginning of the XXI, Physics-Uspekhi, 2004, 47(11):1155. http://dx.doi.org/10.1070/PU2004v047n11ABEH001825

44. Khoruzhy S. Discussions of the internal and external in the practices of self. Moscow Psychotherapeu. J. 2003, 3, pp. 5-25.

45. Kaplan, A. Ya. Harmony of the Big Bang. Domestic Notes. 2014, 2, pp. 123-136.

46. Laplace, P. S. Presentation of the world system. L., Science, Russia. 1982, 376 p.

47. Poincaré, A. On science. M., Science, Russia, 1990, 736 p.

48. Milovich, A. Theory of dynamic interaction of bodies and liquids. M, Russia,1955, $310 \mathrm{p}$.

49. Akhmetov, D.G. Vortex rings. Novosibirsk. Russia, 2007, 151 p., ISBN 978-5-9747-0081-1/

50. Neutrino physics. URL: http://nuclphys.sinp.msu.ru/neutrino/

51. Perkins, D. H., Introduction to High Energy Physics. $4^{\text {th }}$ Edition. CUP, 2001. 426 p.

52. Siegel, E. How The Planck Satellite Forever Changed Our View Of The Universe. 2018.

URL: https://www.forbes.com/sites/startswithabang/2018/07/19/how-the-planck-satellitechanged-our-view-of-the-universe/\#2cb011d77ad2

53. Bucher, M. Physics of the cosmic microwave background anisotropy, 2015, $\underline{\text { arXiv:1501.04288 }}$ [astro-ph.CO], DOI:10.1142/S0218271815300049

54. Kaplan, S.A. Pikelner, S.B. Physics of the interstellar medium. Science, M, 1979, 592 p.

55. Alfven, H.O. G. Plasma universe. Physica Scripta. 1987. 18: pp. 20-28. Plasma cosmology. URL: https://en.wikipedia.org/wiki/Plasma_cosmology\#cite_note-8

56. Longo, M. J. Detection of a dipole in the handedness of spiral galaxies with redshifts z $\sim 0.04$. Physics Letters B, 2011. 699. pp. 224-229. https://doi.org/10.1016/j.physletb.2011.04.008 57. Morrison, D. R. O. Review of Supernova 1987A: Preprint CERN, 26 January, 1988. 58. IceCube Collaboration. Observation of High-Energy Astrophysical Neutrinos in Three Years of IceCube Data // е-принт arXiv:1405.5303.

59. G. Bellini et al., 2010. PLB 687. pp. 299-304. https://doi.org/10.1016/j.physletb.2010.03.051 
60. Vladimirskii, B.M., Bruns, A.V. On presence of false variations of intensity of solar neutrino in radio-chemical experiments. J. Exp. Theor. Phys. 2004. 98(4), pp. 629 - 643

61. Sorokhtin, O. G., Ushakov S. A. Development of the Earth. M: MSU. 2002. 506 p.

62. Pommé, S. et al. Evidence against solar influence on nuclear decay constants, $P L B, \mathbf{2 0 1 6}$, 761, pp. 281-286. https://doi.org/10.1016/j.physletb.2016.08.038

63. Bergeson, S. D., Peatross, J., Ware, M. Precision long-term measurements of beta-decay-rate ratios in a controlled environment, $P L B, \mathbf{2 0 1 7}, 767$, pp. 171-176.

https://doi.org/10.1016/j.physletb.2017.01.030

64. Agostini, M. et al. (Borexino Coll.), Seasonal Modulation of the 7Be Solar Neutrino Rate in Borexino, Astropart.Phys. 2017, 92, pp. 21-29, arXiv:1701.07970.

65. Rivin, Yu. R., Obridko, V. N. Seasonal Variations in Solar High-Energy Neutrino Flux and Their Probable Source, Solar System Research, 2000, 34(6), pp. 501-508.

66. Bellini, G. et al. (Borexino Coll.) Absence of a day-night asymmetry in the 7Be solar neutrino rate in Borexino, Phys. Rev. Lett. 2011, 107, 328 141302; arXiv:1104.1816v1 (hep-ex) 67. Cowan, G. Topics in statistical data analysis for high-energy physics, CERN Yellow Report CERN-2010-002, 2010. pp.197-218, https://arxiv.org/pdf/1012.3589.pdf

68. Douglas, J. H. The Insignificance of Statistical Significance Testing, Journal of Wildlife Management. 1999. 63(3) pp.763-772.

69. Yusupaliev, U. et al. Vortex rings and plasma toroidal vortices in a homogeneous unbounded medium. The maximum path traversed by vortices. Brief reports on physics LPI. 2010 (8). 1-12. 70. Leontovich, M.A. Evolution of the concepts of magnetic and electric lines of force. Sov. Phys. Usp. 1965. 7. pp. 877-880.

71. Mansurov, S.M., Mansurova, L.G. The relationship between the magnetic fields of outer space and the Earth. Geomagnetism and aeronomy. 1971, 11(1), pp. 15-118.

72. Kholmanskiy, A. S. Elementary neutrino physics. New-idea.kulichki.net. 2007. URL: http://new-idea.kulichki.net/pubfiles/190522185738.pdf .

73. Comprehensive measurement of pp-chain solar neutrinos, Nature, 2018, 562, pp. 505-510 doi:10.1038/s41586-018-0624-y] (full text here)

74. Klapdor-Klengrothhouse, G.V. Non-accelerator physics of elementary particles. M, 1997. Russia, 527 p. 
75. Klapdor-Klengrothhouse, G.V., Cyuber, K. Astrophysics of Elementary Particles: M, Russia. 2000. $496 \mathrm{p}$.

76. Kholmanskiy, A.S. Activation of biosystems by an external chiral factor and a decrease in temperature. Asymmetry. 2018, 12(3) pp. 64-77. URL: http://www.cerebralasymmetry.ru/Asymmetry_12_3_2018.pdf; DOI:10.18454/ASY.2018.3.16317

77. Kholmanskiy, A.S. Adaptation of plants to abnormal physical factors. Electron. Math. Med.Biol. J. 2009, 8(3) URL: http://sgma.alpha-design.ru/MMORPH/N-23html/holmanskiy/holmanskiy.htm

78. Avdeeva, L.V. , Coltover, V.K. Nuclear spin catalysis in living nature. Bulletin MSU. Ser. 2. Chemistry. 2016. 57(3) pp. 145-153/ URL: http://www.chem.msu.su/rus/vmgu/163/145.pdf 79. Aksenov, S.I. Water and Its Role in the Regulation of Biological Processes, 2004, 212 p. 80. Kholmanskiy, A. Activation energy of water structural transitions, JMS, 2015, 1089, pp. 124128. DOI: $10.1016 /$ j.molstruc.2015.02.049

81. Kholmanskiy, A. Chirality anomalies of water solutions of saccharides. J. Mol. Liq 2016, 216, pp. 683-687. http://dx.doi.org/10.1016/j.molliq.2016.02.006

82. Pershin, S.M. Ortho/Para $\mathrm{H}_{2} \mathrm{O}$ Conversion in Water and a Jump in Fluidity of Erythrocytes through a Microcapillary at the Temperature $36.6+/-0.3{ }^{\circ} \mathrm{C}$. Phys. Wave Phenomena, 2009, 17(4), pp. 241-250.

83. Casting, D.F. Tun, O.B., Pollack, D.B. How did the climate develop on the terrestrial planets. Sci. Amer. 1988, 4, 32 p.

84. Marchi, S., Black, B.A., Elkins-Tanton, L.T. Massive impact-induced release of carbon and sulfur gases in the early Earth's atmosphere, Earth and Planetary Sci. Let. 2016. 449, pp. 96104. DOI: $\underline{10.1016 / j . e p s l .2016 .05 .032}$

85. Parmon, V.N. Natural selection among molecules. Science First Hand. 2004, 1, pp. 32-41.

86. Giunti C., Studenikin A. Neutrino electromagnetic interactions: a window to new physics. Rev. Mod. Phys. 2015. 87.531. DOI: $\underline{\text { 10.1103/RevModPhys.87.531 }}$

87. Vladimirsky B.M., Temuryants N.A. The effect of solar activity on the biosphere-noosphere. Heliobiology from A.L. Chizhevsky to the present day. M. MNEPU, 2000, 374 p.

88. Chernoschekov K.A., Lepekhin A.V. Materialization of the ideas of A.L. Chizhevsky in epidemiology and microbiology. Tomsk: TSU, 1993. 273 p.

89. Prokofiev, A.A. Seed physiology. M. Science. Russia.1982. 318 p. 
90. Kholmanskiy, A. Dependence of Wheat Seed Germination Kinetics on Temperature and Magnetic Field. Res. J. Seed Sci. 2016, 1, pp. 22-28; DOI: 10.3923/rjss.2016.22.28.

91. Galaziy, G.I. Baikal in questions and answers. Irkutsk, Russia, 1987, 282 p.

92. Takagi, S., Tagawa, M. Predominance of right-handed spirals in human eccrine sweat ducts. Japan. J. Physiol. 1955, 5(2) pp. 122-130.

93. Souman, J. L. Frissen, I. Sreenivasa, M. N. Ernst 3, M.O. Walking Straight into Circles. Current Biology 2009, 19, pp. 1538-1542.

94. Kovalzon, V.M. Foundations of somnology: physiology and neurochemistry of the cycle «wakefulness-sleep». M, Russia, 2014, 239 p.

95. Dean, W., Morgenthaler, J., Fowkes, S. The Melatonin. Chapter Smart Drugs II. 2000. 96. Kholmanskiy, A., Zaytseva, N. Chiral Factor of Circadian Rhythm of Human Physiology, Int. J. Res. in Pharm. Biosci. 2018, 5(4) pp. 6-10. https://www.ijrpb.org/papers/v5-i4/2.pdf 97. Progress in brain research, v. 10. Structure and function of the Epiphyses cerebri, Eds: J. Ariens Kappers and J.P. Schade. L., N. Y. 1965.

98. Lang S. B., Marino A. A., Berkovic G., et al. Piezoelectricity in the human pineal gland. Bioelectrochem. Bioenerg. 1996, 41, pp. 191-195.

99. Afanasyev, Yu. I., Kuznetsov, S. T., Yurina, N. A., et al. Histology, cytology and embryology, M. Med. Russia, 2004. 768 p.

100. Rahnama, M., Bokkon, I., Tuszynski, J. et al, Emission of Biophotons and Neural Activity of the Brain. J. Integr. Neurosci. 2011, 10(1), pp. 65-88.

https://doi.org/10.1142/S0219635211002609.

101. Jonas, J. B., Schneider, U., Gottfried, O.H. Naumann Count and density of human retinal photoreceptors. Graefe's Arch. Clin. Exp. Ophthalmol 1992, 230, pp. 505-510.

102. Belowashkin, A. The temperature rhythm of our body: to understand and use. 2017. URL: https://www.beloveshkin.com/2017/03/temperaturnyj-ritm-nashego-organizma-ponyat-iispolzovat.html.

103. Temuryants, N., Shekhotkin, A., Nasilevich, V. Magnitosensitivity of an epiphysis. Biophys. 1998, 43(5) pp. 761-765.

104. Kholmanskiy, A.S., Minakhin, A.A. Interconnection of electrical oscillations of the heart and brain. Bull. St.-P. State Univ. Med. 2018, 13(2) pp. 117-135. https://dspace.spbu.ru/bitstream/11701/10429/1/01-Kholmansky.pdf 
105. Khelimskiy, A. M. Epiphysis. Medicine, M. Russia, 1969, 183 p.

106. Saveliev, S.V., Erofeeva, E.A., Fokin, E.I. et al. Concrements of the human epiphysis in schizophrenia. Arh. pathology 2004, 4, pp. 13-16.

107. Aleshkevich, V.A., Dedenko, L.G., Karavaev, V.A. Oscillations and waves. MSU, M, Russia, 2001.

108. Bókkon, I. Creative Information. J. Bio. Systems. 2003, 1, pp. 1-17.

https://www.researchgate.net/publication/274201844_Creative_information

109. Kumar, S. et al. Possible existence of optical communication channels in the brain. Sci Rep.

2016; 6: 36508 DOI: $10.1038 / \operatorname{srep} 36508$

110. Zangari, A., Micheli, D., Galeazzi, R., Tozzi. A. Node of Ranvier as an Array of BioNanoantennas for Infrared Communication in Nerve Tissue. Scientific Repots. 2018. 8. 539. 